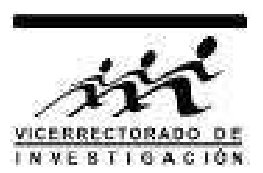

\title{
Estudio de la dinámica del espesor óptico de los aerosoles en Perú y América del Sur a partir de las imágenes MODIS de los satélites TERRA y AQUA en el periodo 2000-2013
}

\author{
Noelia Rojas Benavente* y Joel Rojas Acuña \\ Facultad de Ciencias Físicas, Universidad Nacional Mayor de San Marcos, Lima, Perú
}

Recibido 14 enero 2015 - Aceptado 30 abril 2015

\begin{abstract}
El objetivo de este trabajo es estudiar la dinámica de los aerosoles en el Perú y América del Sur entre las latitudes $4^{\circ} \mathrm{N}-24^{\circ} \mathrm{S}$ y las longitudes $83^{\circ} \mathrm{W}-33^{\circ} \mathrm{W}$, a partir de las imágenes de los promedios mensuales del espesor óptico de los aerosoles, Aerosol Optical Thickness, AOT, en la longitud de onda de $550 \mathrm{~nm}$ medidos por el sensor MODIS, Moderate Resolution Imaging Spectroradiometer, a bordo de los satélites TERRA y AQUA, para un periodo de catorce años, del 2000 al 2013. Se han usado los datos AOT in-situ en los horarios de 6 estaciones AERONET de América del Sur que incluyen cuatro de Brasil, tales como, Rio Branco, Paraná Ji, Alta Foresta y Campo Grande; uno de Chile, la estación de Arica y uno de Bolivia, la estación de La Paz; además incorporamos 166 datos imágenes AOT-MODIS-TERRA de los productos mensuales obtenidos para un periodo del 2000 al 2013 y 138 datos imágenes AOT-MODIS-AQUA de los productos mensuales obtenidos para un periodo del 2002 al 2013 estimados a partir de la herramienta de visulaización y análisis vía Web Giovanni con una resolución de $1^{\circ} \times 1^{\circ}$, es decir, de $110 \mathrm{~km} \times 110 \mathrm{~km}$.

La serie de tiempos AOT-MODIS-TERRA tienen valores máximos y mínimos entre 0.0 y 0.499 y sus valores máximos se ubican en la temporada seca del Hemisferio Sur, entre agosto a noviembre, durante los 14 años. La serie de tiempos AOT-MODIS-AQUA tienen valores máximos y mínimos entre 0.0 y 0.493 y sus valores máximos se ubican en la temporada seca, durante los 12 años. La serie de tiempos AOT-AERONET en las cuatro estaciones de Brasil muestra una variación estacional con máximos en la estación seca durante los 14 años, mientras que, en las estaciones de Chile y Bolivia se muestra una variación estacional muy diferente a los datos AOT/MODIS, teniendo pocos registros de datos AOT. Las gráficas de dispersión de las series de tiempo AOT-MODIS vs AOT-AERONET muestran una alta correlación de 0.8 a 0.9 en cuatro estaciones de Brasil y una correlación baja de 0.33 a 0.39 en la estación de Chile y de 0.13 a 0.19 en la estación de Bolivia.

Palabras claves: Aerosoles, MODIS, espesor óptico de aerosoles, AOT.
\end{abstract}

${ }^{*}$ rnoeliab@gmail.com 
Rev. Inv. Fis. 18, 151801401 (2015)

\title{
Study of the dynamics of aerosol optical thickness in Perú and South America from images MODIS onboard the AQUA and TERRA satellites in $2000-2013$ period
}

\begin{abstract}
The aim of this work is to study the dynamics of aerosols in Peru and South America at latitude $4^{\circ} \mathrm{N}-24^{\circ} \mathrm{S}$ and at longitude $83^{\circ} \mathrm{W}-33^{\circ} \mathrm{W}$, from monthly averages images Aerosol Optical Thickness, $\mathrm{AOT}$, at $550 \mathrm{~nm}$ wavelength measured by the Moderate Resolution Imaging Spectroradiometer, MODIS, sensor onboard the TERRA and AQUA satellites for a period of fourteen years, from 2000 to 2013. The AOT data in-situ in 6 AERONET stations of South America that includes four from Brazil, such as, Rio Branco, Paraná Ji, Alta Foresta, and Campo Grande; from Chile, the Arica station and from Bolivia, La Paz station, and 166 AOT-MODIS-TERRA data images from the monthly products obtained during the period 2000 to 2013, and 138 AOT-MODIS-AQUA data images from the monthly products obtained during the period 2002 to 2013 which was estimated using the Giovanni Web-based online visualization and analysis tool with a $1^{\circ} \times 1^{\circ}$ resolution, i.e., $110 \mathrm{~km} \times 110 \mathrm{~km}$.

The AOT-MODIS-TERRA time series have maximum and minimum values from 0.0 to 0.499 , taking their maximum values in the dry season of South Hemisphere between August to November for 14 years. The AOT-MODIS-AQUA time series have maximum and minimum values from 0.0 to 0.493 , taking their maximum values in the dry season during the 12 years. The AOT-AERONET time series from Brazil four stations shows a seasonal variation with peaks in the dry season for 14 years, meanwhile, from Chile and Bolivia stations shows a very different seasonal variation AOT/MODIS data, having few records of AOT data. The scatter plots of AOT-MODIS-vs-AOT-AERONET time series show a high correlation from 0.8 to 0.9 at four stations in Brazil and low correlation, 0.33-0.39 in the Chile station and 0.13-0.19 in the Bolivia station.
\end{abstract}

Keywords: Aerosols, MODIS, aerosol optical thickness, AOT.

Durante el siglo pasado, hemos sido testigos presenciales de los cambios significativos en el clima de toda la Tierra. Se considera que uno de los principales factores que influyen en estos cambios es el aumento de la concentración de los aerosoles que se encuentran suspendidas en la atmósfera [1]. Los aerosoles son sistemas coloidales de partículas sólidas o líquidas que tienen tamaños menores a un micrómetro y son generados por los procesos naturales de la Tierra como la niebla, las nubes, los geisers, los volcanes y por las actividades antropogénicas como los humos y los polvos de la industria, de los generadores de energía y de los medios de transporte tanto terrestres como aéreos.

Se conjetura que los aerosoles troposféricos influyen significativamente en el cambio climático global, perturbando el balance de la energía radiativa entre la superficie terrestre y la parte superior de la atmósfe$\mathrm{ra}$, mediante el bloqueo de las radiaciones electromagnéticas de onda larga emitidas por la superficie de la Tierra, efecto de calentamiento, y mediante el aumento de la reflectividad de la atmósfera de las radiaciones electromagnéticas que provienen del Sol, efecto de enfriamiento [2]. Asimismo, los aerosoles tienen una gran influencia en el ciclo hidrológico [3], debido a que sus propiedades higroscópicas les permiten actuar como núcleos de condensación de nubes, CCN, que a su vez influyen en los procesos de nube/precipitación, y en el albedo mundial.

La alta variación espacial y temporal de la distribución de tamaño de los aerosoles genera una incertidumbre en la estimación del efecto de los aerosoles en el balance radiativo de la atmósfera, debido a que las propiedades físicas, en este caso las ópticas, de los aerosoles dependen del tamaño de los mismos [4]. A diferencia de los gases de efecto invernadero, los aerosoles no están bien mezclados en la atmósfera. Ellos son espacial y temporalmente no homogéneos, y por lo general, se concentran cerca de la superficie de la Tierra. La altura a la que se encuentran los aerosoles es de 2 a $3 \mathrm{~km}$, mientras que los gases de efecto invernadero se encuentran hasta $8 \mathrm{~km}$ [5].

La concentración de los aerosoles sobre Brasil, se desplaza hacia el Perú por los vientos alisios [6]. Este proceso dinámico de los aerosoles puede ser mejor estudiado por la teledetección de los aerosoles que permite una buena cobertura global para la determinación 
de la distribución espacial y temporal de los aerosoles en varias escalas de resolución. Como los aerosoles son una fuente de absorción de radiacción electromagnética, una propiedad óptica del aerosol, es el espesor óptico, que proporciona información sobre el tamaño de las partículas de aerosol que atenúan la radiación a una longitud de onda específica [7]. Este parámetro se mide mediante un fotómetro en las estaciones terrestres de AERONET [8]. El programa AERONET, tiene como objetivo medir las propiedades ópticas de los aerosoles sobre la superficie terrestre y comparar con los datos AOT obtenidos mediante los satélites. Este programa de la red requiere de la estandarización de la calibración de los instrumentos y del proceso de medición. Por lo tanto, se pueden comparar los datos de las distintas estaciones pertenecientes a la red. Existen 499 estaciones distribuídas en todo el mundo pertenecientes a AERONET. Existen tres niveles de datos: En el nivel 1.0 están los datos sin compensación, en el nivel 1.5 están los datos sin nubes, y en nivel 2.0 están los datos sin nubes y calibrados. Las herramientas de referencia se calibran en el Observatorio M. L. con la técnica de Langley.

En el caso de Perú, no existen estaciones terrestres operacionales pertenecientes a AERONET, la forma de obtener datos sobre el Perú es usando la herramienta de visualización y análisis vía Web Giovanni [9] que proporciona de manera sencilla e intuitiva la visualización, el análisis y el acceso a grandes cantidades de datos científicos de los sensores que observan la Tierra sin tener que descargar los datos originales desde sus servidores [9].

El objetivo de este trabajo es estudiar la dinámica de los aerosoles en el Perú y en América del Sur en las latitudes $4^{\circ} \mathrm{N}-24^{\circ} \mathrm{S}$ y en las longitudes $83^{\circ} \mathrm{W}-33^{\circ} \mathrm{W}$ a partir de las imágenes mensuales del espesor óptico de los aerosoles, AOTs, detectados a $550 \mathrm{~nm}$ de longitud de onda por los sensores MODIS en los satélites TERRA y AQUA. También se usaron los datos obtenidos in-situ en los horarios de seis estaciones de AERONET de las cuales cuatro se ubican en Brasil, Rio Branco, Ji Paraná SE, Alta Foresta y Campo Grande; uno en Chile, la estación de Arica y uno en Bolivia, la estación de La $\mathrm{Paz}$, realizando la comparación con los datos mensuales de los AOTs obtenidos por MODIS los durante catorce años que abarca desde el año 2000 hasta el 2013 [10].

\section{Datos y área de estudio}

El área de estudio corresponde a una parte de América del Sur. El territorio se extiende desde los $4^{\circ}$ latitud norte, en el septentrional de Brasil hasta los $24^{\circ}$ de latitud sur, Figura 1, que corresponde al norte de Chile. Limita por el Este con el océano Atlántico; por el Oeste con el océano Pacífico.

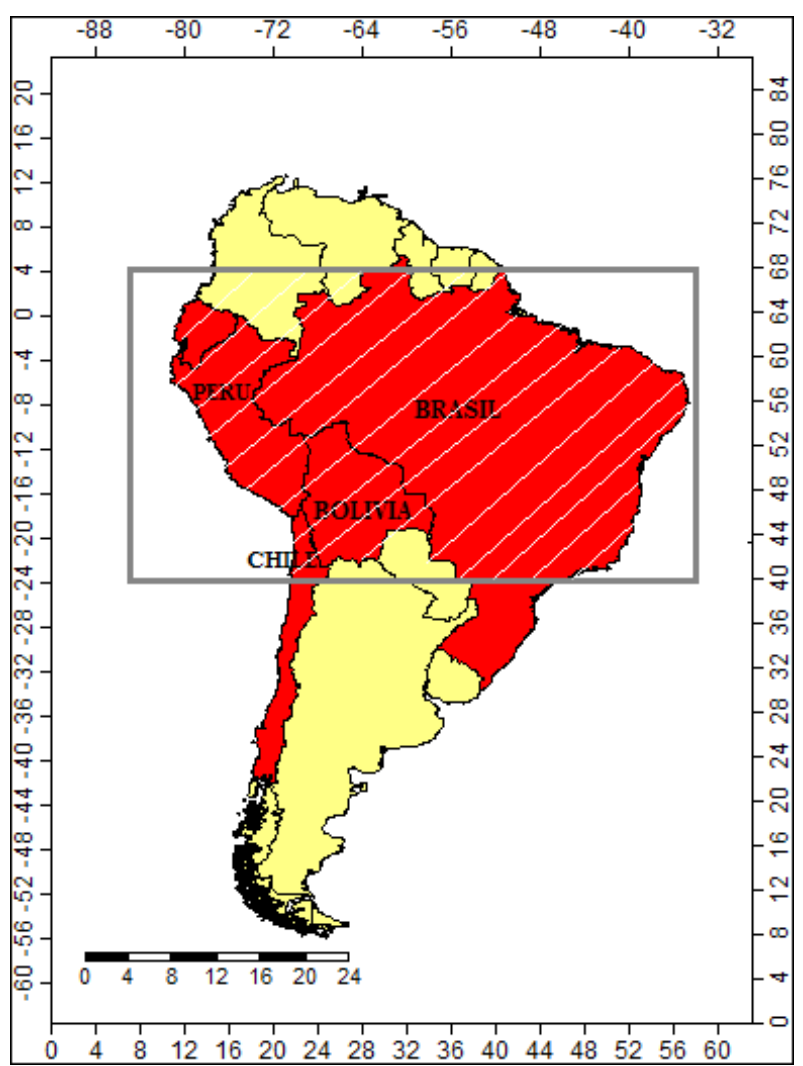

Figura 1: Área de estudio de América del Sur.

\section{Datos espaciales y temporales de los es- pesores ópticos de los aerosoles}

Los datos de los AOTs procedentes del sensor MODIS y de la red AERONET son utilizados en este trabajo. Los datos imágenes de los AOTs de los productos mensuales son del nivel 3, además se han usado 166 datos imágenes AOT procedentes del sensor MODIS abordo del satélite TERRA durante el período del año 2000 al 2013 y 138 datos imágenes AOT de los productos mensuales procedentes del sensor MODIS abordo del satélite AQUA durante el periodo del año 2002 al 2013. La herramienta de visualización y análisis vía Web Giovanni es usado para estimar las imágenes con periodicidad mensual AOT-MODIS-TERRA y AOT-MODIS-AQUA con resolución $1^{\circ} \times 1^{\circ}$, es decir, de $110 \mathrm{~km} \times 110 \mathrm{~km}$ y abarcan un área de aproximadamente $16^{\prime} 940,000 \mathrm{~km}^{2}$. 
También, la herramienta vía Web Giovanni es usado para estimar las series de tiempo AOT-MODIS-TERRA y AOT-MODIS-AQUA en promedios mensuales y en promedios espaciales del área de estudio, los 166 datos AOT para TERRA y los 138 datos AOT para AQUA. Se han utilizado datos AOTs en horarios in-situ de 6 estaciones de AERONET en América del Sur, de los cuales cuatro corresponde a Brasil, Rio Branco, Paraná Ji, Alta Foresta y Campo Grande, uno a Chile, la esta- ción de Arica y uno a Bolivia, la estación de La Paz. En cada estación se ha obtenido los promedios mensuales y se ha generado una serie de tiempo AOT mensual de 14 años que corresponden a 168 datos AOT. La lista de las características del sensor MODIS y de las estaciones AERONET se muestra en la Tabla 1 y Tabla 2. Los datos espaciales y temporales AOT, del sensor MODIS y de las estaciones AERONET, están disponibles al público en la página Web [9].

Tabla 1: Características de las mediciones por satélite

\begin{tabular}{ccccccc}
\hline Satélite & Sensor & $\lambda(\mathrm{nm})$ & $\begin{array}{c}\text { Resolución } \\
\text { espacial }\end{array}$ & $\begin{array}{c}\text { Resolución } \\
\text { temporal }\end{array}$ & Periodo & Región \\
\hline TERRA & MODIS & 550 & $1^{\circ} \times 1^{\circ}$ & mensual & $2000-2012$ & $4^{\circ} \mathrm{N}-24^{\circ} \mathrm{S} ; 83^{\circ}-33^{\circ} \mathrm{W}$ \\
AQUA & MODIS & 550 & $1^{\circ}$ times $1^{\circ}$ & mensual & $2002-2012$ & $4^{\circ} \mathrm{N}-24^{\circ} \mathrm{S} ; 83^{\circ}-33^{\circ} \mathrm{W}$ \\
\hline
\end{tabular}

Tabla 2: Estaciones de AERONET en América del Sur.

\begin{tabular}{llrrrr}
\hline Estación & País & $\begin{array}{c}\text { Latitud } \\
\left({ }^{\circ}\right)\end{array}$ & $\begin{array}{c}\text { Longitud } \\
\left({ }^{\circ}\right)\end{array}$ & $\begin{array}{c}\text { Altura } \\
(\mathrm{m})\end{array}$ & Periodo \\
\hline Rio Branco & Brasil & -9.954 & -67.869 & $212 \mathrm{~m}$ & $2000-2011$ \\
Paraná Ji & Brasil & -10.934 & -62.852 & 218 & $2006-2010$ \\
Alta Foresta & Brasil & -9.871 & -56.104 & 227 & $2000-2012$ \\
Campo Grande & Brasil & -20.438 & -54.538 & 677 & $2003-2011$ \\
Arica & Chile & -18.472 & -70.313 & 25 & $2000-2011$ \\
La Paz & Bolivia & -16.539 & -68.066 & 3439 & $2006-2011$ \\
\hline
\end{tabular}

\section{Determinación del AOT a partir de las imágenes MODIS-AQUA y MODIS-TERRA}

Existen numerosas técnicas para observar y cuantificar las propiedades físicas y químicas de los aerosoles, ya sean in-situ o por teledetección [11]. Combinando las mediciones desde la superficie del suelo, desde el aire y el espacio, estos proporcionan los perfiles de las propiedades de los aerosoles tales como la carga, la distribución del tamaño y la composición química. Otras técnicas in-situ se basan en mediciones ópticas y miden la dispersión del aerosol dentro de una cavidad para determinar los coeficientes de extinción y retro dispersión, back-scattering, medidos por los nefelómetros [12]. En la teledetección, los sensores observan un campo de radiación a medida que interactúa con la atmósfera y la superficie. Los radiómetros también pueden estar a bordo de los satélites en órbita, tal como ocurre con el MODIS-TERRA, para recuperar la información contínua sobre las escalas regionales y mundial [13-15].
Por otro lado, existen numerosos métodos para el análisis de los datos de los aerosoles obtenidos por las imágenes MODIS, en este trabajo mencionamos dos. El primero es la recuperación de las propiedades de los aerosoles sobre el suelo a partir de la inversión de la reflectancia espectral observada por el sensor MODIS usando un Algoritmo Operacional de Segunda Generación y el segundo, se realiza mediante el Algoritmo Simplificado para la recuperación las propiedades de los aerosoles de las imágenes MODIS.

\section{A. Algoritmo Operacional de Segunda Generación}

Para la recuperación de las propiedades de los aerosoles sobre el suelo a partir de la inversión de la reflectancia espectral observada por el sensor MODIS.

Desde comienzos del año 2000, la recuperación cuantitativa, global y operacional de las propiedades de los aerosoles sobre el suelo se han realizado a partir de la reflectancia de la superficie observada por el MODIS. 
Esos productos han sido evaluados y validados contínuamente y en algunas oportunidades se han mejorado. Este algoritmo operacional de segunda generación ejecuta una inversión simultánea de los dos canales visibles con $0.47 \mu \mathrm{m}$ y $0.66 \mu \mathrm{m}$ y un canal del infrarrojo de onda corta a $2.12 \mu \mathrm{m}$. La inversión de los tres canales produce tres parámetros aproximadamente independientes, el espesor óptico de los aerosoles $\tau$ en $0.55 \mu \mathrm{m}$, la ponderación fina o sin polvo $\eta$, y la reflectancia de la superficie en $2.12 \mu \mathrm{m}$. Las validaciones preliminares de este algoritmo muestran muchas mejoras en la recuperación de $\tau$, donde la regresión MODIS/AERONET de $\tau$ en 0.55 $\mu \mathrm{m}$ está dado por $y=1.01 x+0.03$, y $R=0.90$. Luego, la reflectancia en la cima de la atmosfera $\rho_{\lambda}$ está dado por

$$
\begin{aligned}
\rho_{\lambda}^{*}\left(\theta_{0}, \theta, \phi\right)=\rho_{\lambda}^{a}\left(\theta_{0}, \theta, \phi\right)+ & \\
& \frac{F_{\lambda}\left(\theta_{0}\right) T_{\lambda}(\theta) \rho_{\lambda}^{s}\left(\theta_{0}, \theta, \phi\right)}{1-s_{\lambda} \rho_{\lambda}^{s}\left(\theta_{0}, \theta, \phi\right)}
\end{aligned}
$$

donde $\rho_{\lambda}^{s}$ es la "reflectancia de camino" atmosférica, $F_{\lambda}$ es el flujo hacia abajo normalizado para la reflectancia de la superficie cero, $T_{\lambda}$ representa la transmisión total hacia arriba en el campo de visión del satélite, $s_{\lambda}$ es el cociente de retrodispersión atmosférica, y $\rho_{\lambda}^{s}$ es la reflectancia de la superficie angular. Estas son funciones del ángulo cenit del Sol $\theta_{0}$, el ángulo cenit del satélite $\theta$ y el ángulo acimut relativo Sol/satélite $\phi$. Excepto para la reflectancia de la superficie, cada termino del lado de la mano derecha de la ecuación (11) es una función de la dispersión de Rayleigh, tipo de aerosol y carga de aerosol $(\tau)$. Mientras que la ecuación (11) es técnicamente válida para una superficie Lambertiana uniforme, las propiedades bidireccionales de la reflectancia de la superficie puede ser aproximado por el valor de la reflectancia de la superficie según la geometría de visión del satélite y del Sol. Asumiendo que un pequeño conjunto de tipo y carga de aerosoles puede describir el rango de aerosoles global, el algoritmo cuenta con un Look Up Table, LUT, que contiene simulaciones precalculadas de esas condiciones de los aerosoles. El objetivo del algoritmo es examinar el LUT para determinar las condiciones que mejor simulan la reflectancia espectral observada por el MODIS, $\rho_{\lambda}^{m}$, y la recuperación de las propiedades de los aerosoles que incluyen $\tau$ y $\eta$.

La dificultad radica en hacer las suposiciones más apropiadas acerca de las contribuciones de la superficie y la atmosfera. Con el fin de justificar el uso de los productos de aerosoles del MODIS para cualquier aplicación, la incertidumbre de la recuperación debe ser cuantificada. Antes del lanzamiento, las pruebas de sen- sibilidad y las simulaciones en aviones sugirieron que el MODIS debe ser capaz de recuperar $\tau$ dentro del $20 \%$ al $30 \%$ como error esperado sobre muchas superficies del suelo vegetadas y semi-vegetadas. Desde el lanzamiento, los estudios de validación sugieren que el error esperado sobre el suelo puede ser representado por

$$
\Delta \tau= \pm 0.05 \pm 0.15 \tau \text {. }
$$

En la longitud de onda de $0.55 \mu \mathrm{m}$, se puede considerar una regresión MODIS / AERONET representativo de $\tau_{0.55}$ sobre el suelo como

$$
\tau_{\text {MODIS }}=0.1+0.9 \tau_{\text {AERONET }} .
$$

Cuando la reflectancia de la superficie está presente, el segundo término en ecuación (11) es diferente de cero. El flujo es una función solo de la atmófera, mientras que, los términos de retrodispersión atmosférica $s$ y de transmisión $T$ son funciones de la atmósfera y de la superficie. Por lo tanto, el RT3 es corrido dos veces con distintos valores positivos de la reflectancia de la superficie

$$
s=\frac{1}{\rho_{1}^{s}}\left[1-\frac{F_{d} T \rho_{1}^{s}}{\rho^{*}-\rho^{a}}\right] \quad s=\frac{1}{\rho_{2}^{s}}\left[1-\frac{F_{d} T \rho_{2}^{s}}{\rho^{*}-\rho^{a}}\right] .
$$

Aquí, se escoge los valores de 0.1 y 0.25 para las reflectancia de la superficie, $\rho_{1}^{s}$ y $\rho_{2}^{s}$. Esas dos ecuaciones son resueltas para las dos incógnitas $s$ y $T$. Estos valores están dentro del LUT, para cada índice $\tau$, longitud de onda y modelo de aerosoles. La relación de $0.47 \mu \mathrm{m}$ a $0.66 \mu \mathrm{m}$ es mayor que 0.47 vs 2.12 porque tiene una alta correlación, $R=0.87$ y una menor dispersión,

$$
\begin{aligned}
& \rho_{0.66}^{s}=f\left(\rho_{2.12}^{s}\right) \\
& \rho_{0.47}^{s}=g\left(\rho_{0.66}^{s}\right)
\end{aligned}
$$

donde $f()$ y $g()$ son diferentes relaciones. Excepto para áreas urbanas, muchas superficies parecen tener una relación de reflectancia de la superficie VIS vs 2.12 que puede estar relacionada a un índice de vegetación, VI.

La bien conocida NDVI definida como una función del rojo, a $0.66 \mu \mathrm{m}$ en el canal 1 e infrarrojo cercano a $0.86 \mu \mathrm{m}$ en el canal 2, son influídas por el aerosol, negando su utilidad para determinar el tipo de superficie. Intentamos trabajar con otro VI que tenga diferente sensibilidad para condiciones atmosféricas para el aerosol y encontramos el más prometedor $\mathrm{NDVI}_{\text {swir }}$ definido como

$$
\mathrm{NVDI}_{\text {swir }}=\frac{\rho_{1.24}^{m}-\rho_{2.12}^{m}}{\rho_{1.24}^{m}+\rho_{2.12}^{m}}
$$


donde $\rho_{1.24}^{m}$ y $\rho_{2.12}^{m}$ son las reflectancias medidas por el MODIS del canal 5 a $1.24 \mu \mathrm{m}$ y el canal 7 a $2.12 \mu \mathrm{m}$. Estas longitudes de onda más grandes son mucho menos influídos por el aerosol, a excepción de los aerosoles pesados y polvo, luego son potencialmente más útiles para estimar las condiciones de superficie. En condiciones libre de aerosoles el $\mathrm{NDVI}_{\text {swir }}$ es altamente correlacionado con el NDVI regular. Un valor de $\mathrm{NDVI}_{\text {swir }}>0.6$ es relativo a la vegetación más abundante, mientras que $\mathrm{NDVI}_{\text {swir }}<0.2$ es representativo de una vegetación en estado latente y escasa.

\section{Parametrización final de la relación de la reflectan- cia de la superficie VISvs2.12}

Los resultados del ejercicio de corrección atmosférica global implican que no solo la relación de la superficie VIS vs 2.12 difiere de los cocientes asumidos por el algoritmo $\mathrm{C004}$, ellos también tienen una fuerte dependencia de la geometría y el tipo de superficie. La relación de la reflectancia de la superficie VISvs2.12 es parametrizada como una función de $\mathrm{NDVI}_{\text {swir }}$ y el ángulo de dispersión $\theta$, tal que la ecuación (5) puede ser expandido como

$$
\begin{aligned}
& \rho_{0.66}^{s}=\rho_{2.12}^{2} \cdot \text { slope }_{0.66 / 2.12}+\text { yint }_{0.66 / 2.12} \\
& \rho_{0.47}^{s}=\rho_{0.66}^{2} \cdot \text { slope }_{0.47 / 0.66}+\text { yint }_{0.47 / 0.66}
\end{aligned}
$$

donde

$$
\begin{aligned}
\text { slope }_{0.66 / 2.12} & =\text { slope }_{0.66 / 2.12}^{\mathrm{NDVI}_{\text {swir }}}+0.002 \theta-0.27 \\
\text { yint }_{0.47 / 0.66} & =-0.00025 \theta+0.033 \\
\text { slope }_{0.47 / 0.66} & =0.49 \mathrm{y} \\
\text { yint }_{0.47 / 0.66} & =0.005
\end{aligned}
$$

donde en cambio

$$
\begin{gathered}
\text { slope }_{0.66 / 2.12}^{\mathrm{NDVI}_{\text {swir }}}=0.48 ; \quad \mathrm{NDVI}_{\text {swir }}<0.25, \\
\text { slope }_{0.66 / 2.12}^{\mathrm{NDVI}_{\text {swir }}}=0.58 ; \quad \mathrm{NDVI}_{\text {swir }}<0.75, \\
\text { slope }_{0.66 / 2.12}^{\mathrm{NDVI}_{\text {swir }}}=0.48+0.2\left(\mathrm{NDVI}_{\text {swir }}-0.25\right) \\
0.25 \leq \mathrm{NDVI}_{\text {swir }} \leq 0.75
\end{gathered}
$$

Podemos considerar la relación descrita por la Figura 1 del artículo de Levy et al [16] como la relación promedio global y la parametrización para describir las perturbaciones para el ángulo y tipo de suelo. Nótase que mientras, la parametrización de arriba en las ecuaciones (8)-(10) estuvo basada en los resultados de las
Figuras 1-3 del artículo de Levy et al, los coeficientes no son idénticos a aquellos mostrados en las figuras de Levy [16]. El conjunto de datos corregidos atmosféricamente es la representación más ancha y más comprensiva de las relaciones de las reflectancias de las superficies globales, y aún es limitado a las posiciones de los sitios AERONET, que en cambio son mayormente concentradas en ciertas regiones geográficas. La prueba y error fue usado para modificar los resultados básicos de la corrección atmosférica basada en AERONET, para dar las recuperaciones basadas en AERONET globalmente, especialmente en aquellos lugares en los no existen o existen pocas estaciones de AERONET.

Esperamos que la parametrización deduzca estimaciones más exactas de la reflectancia de la superficie en promedio que aquellas estimadas usando cocientes fijos. Reescribiendo la ecuación (11) pero notando que la reflectancia total espectral calculada $\rho_{\lambda}^{*}$ en la cima de la atmósfera es la suma ponderada de la reflectancia espectral de una combinación de modelos de aerosoles fina y gruesa, escrita como

$$
\rho_{\lambda}^{*}=\eta \rho_{\lambda}^{* f}+(1-\eta) \rho_{\lambda}^{* C}
$$

donde $\rho_{\lambda}^{* f}$ y $\rho_{\lambda}^{* C}$ son cada uno compuestos de la reflectancia de la superficie $\rho_{\lambda}^{s}$ y de la reflectancia del camino atmosférico de los modelos de aerosoles separados. Esto es,

$$
\begin{aligned}
\rho_{\lambda}^{* f} & =\rho_{\lambda}^{a f}+\frac{F_{d \lambda}^{f} T_{\lambda}^{f} \rho_{\lambda}^{s}}{\left(1-s_{\lambda}^{f}\right) \rho_{\lambda}^{s}} \quad \mathrm{y} \\
\rho_{\lambda}^{* C} & =\rho_{\lambda}^{a C}+\frac{F_{d \lambda}^{C} T_{\lambda}^{C} \rho_{\lambda}^{s}}{\left(1-s_{\lambda}^{C}\right) \rho_{\lambda}^{s}}
\end{aligned}
$$

donde $\rho_{\lambda}^{a f}$ y $\rho_{\lambda}^{a C}$ son las reflectancias de camino atmosférica de modelo fino y grueso; $F_{d \lambda}^{f}$ y $F_{d \lambda}^{C}$ son los flujos hacia abajo normalizados para la reflectancia de superficie cero; $T_{\lambda}^{f}$ y $T_{\lambda}^{C}$ representa la transmisión total hacia arriba en el campo de visión del satélite; y $s_{\lambda}$ y $s_{\lambda}^{C}$ son los cocientes de retrodispersión atmosférica. El parámetro de ponderación $\eta$ en la ecuación (10) es definido por $\lambda=0.55 \mu \mathrm{m}$. Este parámetro representa la fracción del espesor óptico total en $0.55 \mu \mathrm{m}$ contribuído por el aerosol fino, sin polvo.

\section{Fracción del espesor óptico de modo fino}

Uno de los productos más importantes producidos por el algoritmo es el cociente del espesor óptico de modo fino al espesor óptico total, o simplemente la fracción de modo fino. Aquí mostramos que esta fracción 
en $0.55 \mu \mathrm{m}$ es el mismo parámetro como eta, el parámetro de ponderación de la reflectancia. Comenzamos con la ecuación siguiente,

$$
\rho_{\lambda}^{L U T}\left(\tau_{\lambda}^{\text {tot }}\right)=\eta \rho_{\lambda}^{f}\left(\tau_{\lambda}^{\text {tot }}\right)+(1-\eta) \rho_{\lambda}^{C}\left(\tau_{\lambda}^{\text {tot }}\right)
$$

donde $\rho_{\lambda}^{f}$ y $\rho_{\lambda}^{C}$ son las reflectancias espectrales totales de modo fino y modo grueso para los mismos espesores ópticos como la reflectancia espectral total $\rho_{\lambda}^{L U T}$, respectivamente, y eta es el parámetro de ponderación de la reflectancia. Nótase que $\rho_{\lambda}^{L U T} \neq \rho_{\lambda}^{f}+\rho_{\lambda}^{C}$ y que las reflectancias total y las componentes son todas funciones del espesor óptico total $\tau_{\lambda}^{t o t}$, y no de las componentes del espesor óptico $\tau_{\lambda}^{f}$ y $\tau_{\lambda}^{C}$.

Todos los espesores ópticos son definidos en 0.55 $\mu \mathrm{m}$. Esto es, por definición al construir el look up table, definimos el espesor óptico total $\tau_{\lambda}^{\text {tot }}$ igual a la suma de las componentes fina $\tau_{\lambda}^{f}$ y gruesa $\tau_{\lambda}^{C}$. Usando la aproximación de dispersión simple,

$$
\begin{gathered}
\rho_{\lambda}^{f}=C \tau_{0.55}^{t o t} P_{\lambda}^{f}, \quad \rho_{\lambda}^{C}=C \tau_{0.55}^{t o t} P_{\lambda}^{C} \quad \mathrm{y} \\
\rho_{\lambda}^{L U T}=C\left(\tau_{0.55}^{f} P_{\lambda}^{f}+\tau_{0.55}^{C} P_{\lambda}^{C}\right)
\end{gathered}
$$

donde $C$ es una constante que depende de la geometría $P_{\lambda}^{f}$ y $P_{\lambda}^{C}$ son las funciones fase de modo fino y modo grueso calculadas para el look up table, respectivamente. No hay $P_{\lambda}^{\text {tot }}$ porque las funciones fase en el look up table son calculadas para la colección de modos fino y grueso individual, no para cualquier distribución de tamaño de aerosol "total". Resolviendo para $\eta$ en la ecuación (13) obtenemos

$$
\eta=\frac{\rho^{L U T}-\rho_{\lambda}^{C}}{\rho_{\lambda}^{f}-\rho_{\lambda}^{C}} .
$$

Sustituyendo las Ecs. (13), (14) y (15) en la Ec. (16) obtenemos

$$
\eta=\frac{c\left(\tau_{0.55}^{f} P_{\lambda}^{f}+\tau_{0.55}^{C} P_{\lambda}^{C}\right)-C \tau_{0.55}^{t o t} P_{\lambda}^{C}}{C \tau_{0.55}^{t o t} P_{\lambda}^{f}-C \tau_{0.55}^{t o t} P_{\lambda}^{C}} .
$$

Eliminando la constante $C$ y usando la definición de $\tau_{0.55}^{\text {tot }}=\tau_{0.55}^{f}+\tau_{0.55}^{C}$ resulta

$$
\begin{aligned}
\eta & =\frac{\left(\tau_{0.55}^{f} P_{\lambda}^{f}+\tau_{0.55}^{C} P_{\lambda}^{C}\right)-\left(\tau_{0.55}^{f}+\tau_{0.55}^{C}\right) P_{\lambda}^{C}}{\tau_{0.55}^{t o t}\left(P_{\lambda}^{f}-P_{\lambda}^{C}\right)} \\
\eta & =\frac{\tau_{0.55}^{f}\left(P_{\lambda}^{f}-P_{\lambda}^{C}\right)}{\tau_{0.55}^{t o t}\left(P_{\lambda}^{f}-P_{\lambda}^{C}\right)} \mathrm{y} \\
\eta & =\frac{\tau_{0.55}^{f}}{\tau_{0.55}^{t o t}}
\end{aligned}
$$

Así el factor de ponderación de la reflectancia eta, es también el cociente entre el espesor óptico de modo fino y el total en $0.55 \mu \mathrm{m}$, como fue definido dentro de los parámetros de la inversión. Nótase que en algunos términos, la dependencia es angular y de $\tau$, vea$\operatorname{mos} \rho^{a}=\rho^{a}\left(\tau, \theta_{0}, \phi\right), F=F\left(\tau, \omega_{0}\right), T=T(\tau, \omega)$, $s=s(\tau)$ y $\rho^{s}=\rho^{s}\left(\tau, \theta_{0}, \phi\right)$, mientras que los otros términos son una función de las propiedades de los aerosoles y no de la cantidad de aerosoles o de la geometría de los mismos. En términos prácticos, parametrizamos la reflectancia de la superficie usando la relación de la reflectancia de la superficie VISvs2.12, que suponemos es una función del ángulo de dispersión y del índice de vegetación.

\section{Selección de los pixeles oscuros}

La Figura 2 ilustra los principales pasos de nuestro nuevo algoritmo del suelo. Cada escena MODIS individual, llamado un granulo, consiste de una franja de datos de 5 minutos, midiendo aproximadamente 1340 $\mathrm{km}$ por $2030 \mathrm{~km}$. Los datos relevantes Level 1 B (L1B) incluye la reflectancia espectral calibrada en ocho bandas de longitud de onda en una variedad de resoluciones espaciales, así como la información de geolocalización asociada. Los datos espectrales incluyen los canales 1 y 2 en $0.66 \mu \mathrm{m}$ y $0.86 \mu \mathrm{m}$ del MODIS en $250 \mathrm{~m}$ de resolución, respectivamente; las longitudes de onda 0.47 , $0.55,1.24,1.64$ y $2.12 \mu \mathrm{m}$ en los canales $3,4,5,6$ y 7 en $500 \mathrm{~m}$, respectivamente y la longitud de $1.38 \mu \mathrm{m}$ en el canal 26 en $1 \mathrm{~km}$. Los datos de geolocalización están en $1 \mathrm{~km}$ e incluye los ángulos $\left(\theta_{0}, \theta, \phi, \theta\right)$, latitud, longitud, elevación y la fecha. Los valores de reflectancia L1B son corregidas para vapor de agua, ozono y dióxido de carbón [16] antes de proceder.

El primer paso es organizar las reflectancias medidas en cajas nominales de $10 \mathrm{~km}$ por $10 \mathrm{~km}$, que corresponden a $20 \times 20$ o $40 \times 40$ pixeles, dependiendo de los canales. Los 400 pixeles en la caja son evaluados píxel por píxel para identificar si el píxel es adecuado para la recuperación de los aerosoles. Nubes, nieve/hielo y cuerpos de agua en suelo, vía pruebas de NDVI, son consideradas no adecuadas y son descartadas. Los pixeles no enmascarados son chequeados por sus brillos. Los pixeles que tienen medidas de reflectancia de 2.12 $\mu \mathrm{m}$ entre 0.01 y 0.25 son agrupadas y clasificadas por su reflectancia de $0.66 \mu \mathrm{m}$. Los más brillantes en 0.66 $\mu \mathrm{m}, 50 \%$ y los más oscuros $20 \%$ son descartadas, con el fin de reducir las nubes y la contaminación de superficie y escala hacia objetos más oscuros en las longitud de onda visibles. Si hay al menos 12 pixeles restantes, 
$10 \%$ de $30 \%$ del original 400 , entonces la reflectancia en cada canal es ponderada, produciendo la reflectancia espectral medida por el MODIS $\rho_{0.47}^{m}, \rho_{0.66}^{m}, \rho_{2.12}^{m}$ y $\rho_{1.24}^{m}$. Estos valores de reflectancia son usados para el Procedimiento A. Si hay menos de 12 pixeles entonces permanecen, por lo tanto, se sigue el Procedimiento B.

\section{Corrigiendo el LUT para elevación}

Se ha considerado en este algoritmo la corrección para los objetos de superficies elevadas. El espesor óptico de Rayleigh a nivel del mar, ROD, $\tau_{R, \lambda}$ en una longitud de onda $\lambda(\mu \mathrm{m})$ puede ser aproximado en el rango visible por

$$
\tau_{R, \lambda}=-0.00877 \lambda^{-4.05}
$$

Cuando no está en el nivel del mar, presión $=1013$ $\mathrm{mb}$, el ROD es función de la presión o altura z, así que puede ser aproximado por

$$
\tau_{R, \lambda}(z=Z)=\tau_{R, \lambda}(z=0) \exp \left[-\frac{Z}{8.5}\right],
$$

donde $Z$ es la altura, en kilómetros, del objeto en la superficie y $8.5 \mathrm{~km}$ es la escala de altura exponencial de la atmósfera. La diferencia entre ROD en $z=0 \mathrm{y}$ $z=Z$ es $\Delta \tau_{R, \lambda}$.

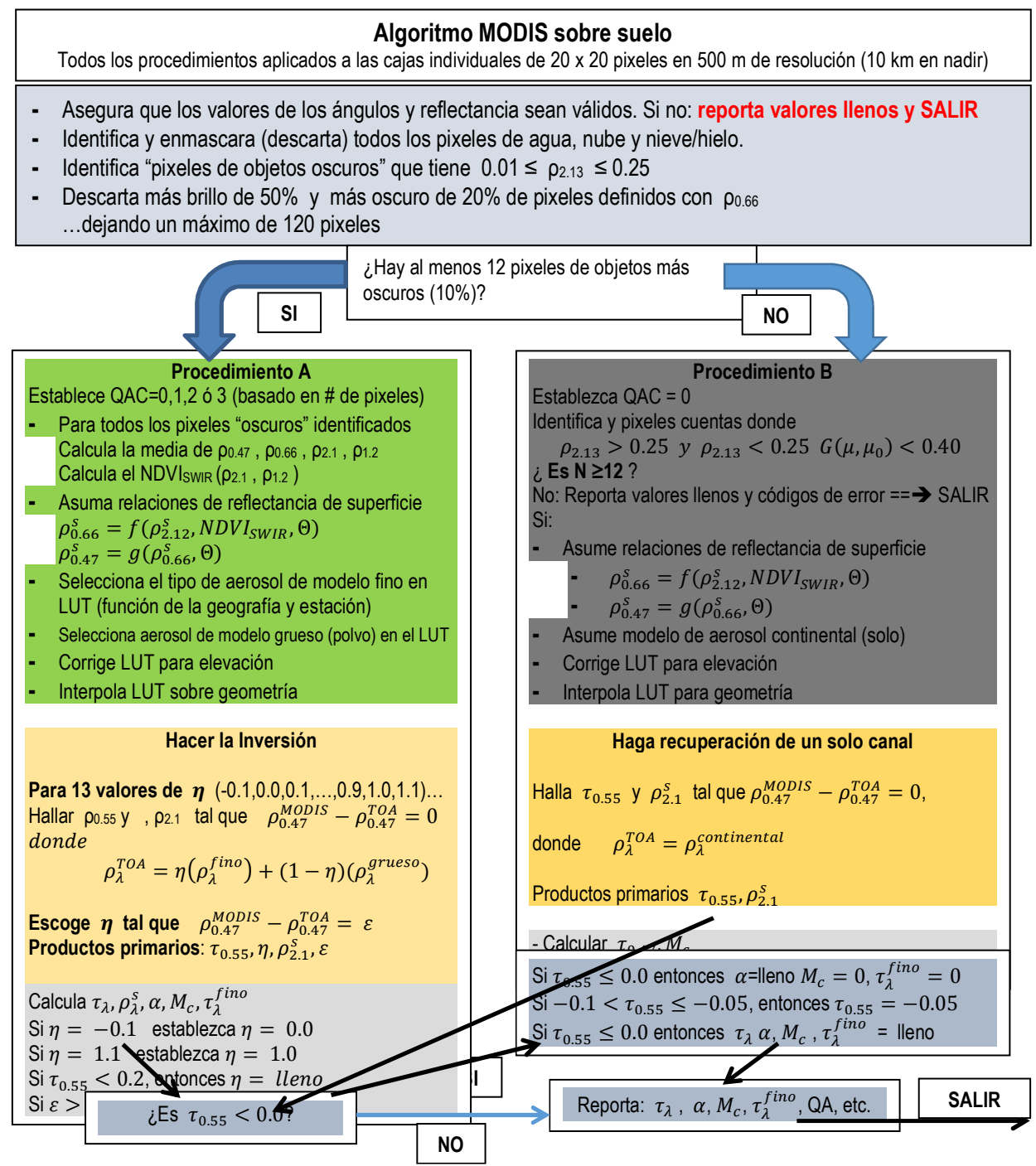

Figura 2: Diagrama de flujo que ilustra la deducción del aerosol sobre el suelo para C005 [16] 


\section{Procedimiento A: Inversión para superficies oscu- ras}

Si se sigue el procedimiento A, para superficies oscuras, el QAC es inicialmente establecido a un valor entre 0 , calidad mala, y 3 , calidad buena, dependiendo del número de pixeles oscuros que permanecen. En el procedimiento $A$, el algoritmo asigna el modelo de aerosoles fino, basado en la posición y el tiempo. Según el LUT, $\rho^{a}, F, T$ y $s$, para el modelo fino y el modelo grueso por separado, son interpolados para ángulos $\left(\theta_{0}, \theta, \phi\right)$, resultando en seis valores para cada parámetro, cada uno de las cuales corresponde a una carga de aerosol diferente, indexado por $\tau$ en $0.55 \mu \mathrm{m}$. La reflectancia de camino de $2.12 \mu \mathrm{m}$ es una función no despreciable de $\tau$, por ejemplo, para el modelo de aerosoles que no absorben, $\tau_{0.55}=0.5$, que corresponde a $\tau_{2.12}=0.055 \mu \mathrm{m}$, así la reflectancia de la superficie es, por lo tanto, también una función de $\tau$. Para valores discretos de $\eta$ entre -0.1 y 1.1 a intervalos de 0.1 , el algoritmo intenta hallar $\tau$ en $0.55 \mu \mathrm{m}$ y la reflectancia de la superficie en $2.12 \mu \mathrm{m}$ que exactamente coincide con la reflectancia en $0.47 \mu \mathrm{m}$ medida por el MODIS. Habrá algún error, $\varepsilon$, en $0.66 \mu \mathrm{m}$. La solución está en el ajuste del error en $0.66 \mu \mathrm{m}$ que es minimizado. En otras palabras,

$$
\begin{aligned}
& \operatorname{ABS}\left(\frac{\rho_{0.47}^{*}-\rho_{0.47}^{m}}{\rho_{0.47}^{m}}\right)=0 \\
& \operatorname{ABS}\left(\frac{\rho_{0.66}^{*}-\rho_{0.66}^{m}}{\rho_{0.66}^{m}}\right)=\varepsilon \\
& \operatorname{ABS}\left(\frac{\rho_{2.12}^{*}-\rho_{2.12}^{m}}{\rho_{2.12}^{m}}\right)=0
\end{aligned}
$$

donde

$$
\begin{aligned}
\rho_{2.12}^{*} & =\eta\left(\rho_{2.12}^{f a}+\frac{F_{d, 2.12}^{f} T_{2.12}^{f} \rho_{2.12}^{f}}{1-s_{2.12}^{f} \rho_{2.12}^{s}}\right) \\
& +(1-\eta)\left(\rho_{2.12}^{C a}+\frac{F_{d, 2.12}^{C} T_{2.12}^{C} \rho_{2.12}^{s}}{1-s_{2.12}^{C} \rho_{2.12}^{s}}\right) \\
\rho_{0.66}^{*} & =\eta\left(\rho_{0.66}^{f a}+\frac{F_{d, 0.66}^{f} T_{0.66}^{f} f\left(\rho_{2.12}^{s}\right)}{1-s_{0.66}^{f} f\left(\rho_{2.12}^{s}\right)}\right) \\
& +(1-\eta)\left(\rho_{2.12}^{C a}+\frac{F_{d, 2.12}^{C} T_{2.12}^{C} \rho_{2.12}^{s}}{1-s_{2.12}^{C} \rho_{2.12}^{s}}\right)
\end{aligned}
$$

$$
\begin{gathered}
\rho_{0.47}^{*}=\eta\left(\rho_{0.47}^{f a}+\frac{F_{d, 0.47}^{f} T_{0.47}^{f} g\left(\rho_{0.66}^{f}\right)}{1-s_{0.47}^{f} g\left(\rho_{0.66}^{s}\right)}\right) \\
+(1-\eta)\left(\rho_{0.47}^{C a}+\frac{F_{d, 0.47}^{C} T_{d, 0.47}^{C} g\left(\rho_{0.66}^{s}\right)}{1-s_{0.47}^{C} \rho_{0.66}^{s}}\right)
\end{gathered}
$$

donde $\rho^{a}=\rho^{a}(\tau), F=F(\tau), T=T(\tau)$ y $s=s(\tau)$ son funciones de $\tau$ y de los índices en el LUT que es calculado por separado para modelos finos y gruesos. $f\left(\rho_{2.12}^{s}\right)$ y $g\left(\rho_{0.66}^{s}\right)$ son descritas por las ecuaciones (8)(10). Nótase que para valores no físicos de $\eta$ son probados, -0.1 y 1.1 , para permitir la posibilidad de suposiciones inapropiadas en uno y otro modelo del aerosol o de la reflectancia de la superficie. Otra vez, los productos primarios son $\tau_{0.55}, \eta_{0.55}$ y la reflectancia de la superficie $\rho_{2.12}^{S}$. El error del ajuste $\varepsilon$ es también notado.

\section{Procedimiento B: Recuperación alternativa para superficies más brillantes}

La determinación de las propiedades de los aerosoles es posible cuando la reflectancia en $2.12 \mu \mathrm{m}$ es más brillante que 0.25 , pero se espera que sea menos exacto, debido a los crecientes errores en la relación VISvs2.12. Sin embargo, si el procedimiento $A$ no fue posible, pero hay menos de 12 pixeles que no son agua y libre de nubes satisfacen lo siguiente

$$
0.25<\rho_{2.12}^{s}<0.25 G<0.40
$$

donde

$$
G=0.5\left(\frac{1}{\mu}+\frac{1}{\sqrt{\mu_{0}}}\right) .
$$

Luego se intenta el procedimiento B. La ecuación (19) es una representación del camino inclinado de la radiación. El concepto es que los ángulos oblícuos, cuando el camino del fotón aumenta, origina más y más señales en la atmósfera y menos desde el suelo. La contribución de la reflectancia de la superficie se hace menos importante y la recuperación puede tolerar mayores valores de la reflectancia desde la superficie. En el procedimiento B, el QAC es automáticamente establecido a 0, "calidad mala".

\section{B. Algoritmo simplificado para la recupe- ración de los aerosoles de imágenes $\mathrm{MO}$ - DIS}

Un algoritmo simplificado para la recuperación de los aerosoles, del inglés Simplified Aerosol Retrieval Algorithm, SARA, ha sido desarrollado sin usar la técnica 
común de construir un Look Up Table, LUT, del Modelo de Transferencia Radiativa, MTR [17]. La justificación de la propuesta del algoritmo de la recuperación de aerosol es determinada por la reflectancia del aerosol descompuesto por la reflectancia en la cima de la atmósfera, TOA, desde la reflectancia de la superficie y la reflectancia del camino de Rayleigh. La reflectancia TOA $\rho_{\text {TOA }}\left(\lambda, \theta_{S}, \theta_{v}, \phi\right)$ es función de los ángulos cenit y acimut del Sol y visión, y puede ser estimado usando la siguiente ecuación [18, 19],

$$
\begin{gathered}
\rho_{\mathrm{TOA}}\left(\lambda, \theta_{S}, \theta_{v}, \phi\right)=\rho_{\text {Aer }}\left(\lambda, \theta_{S}, \theta_{v}, \phi\right)+ \\
\rho_{\text {Ray }}\left(\lambda, \theta_{S}, \theta_{v}, \phi\right)+\frac{T\left(\theta_{S}\right) T\left(\theta_{v}\right) \rho_{\text {sup }}\left(\theta_{S}, \theta_{v}, \phi\right)}{1-\rho_{\text {sup }} S(\lambda)}
\end{gathered}
$$

donde $\theta_{S}$ es el ángulo cenit del sol, $\theta_{v}$ es el ángulo cenit de visión, $\phi$ es el ángulo acimut relativo, $\rho_{\text {Aer }}\left(\lambda, \theta_{S}, \theta_{v}, \phi\right)$ es la reflectancia de los aerosoles que resulta de la dispersión múltiple en la ausencia de las moléculas, $\rho_{\text {Ray }}\left(\lambda, \theta_{S}, \theta_{v}, \phi\right)$ es la reflectancia Rayleigh que resulta de dispersión múltiple en la ausencia de aerosoles, $T\left(\theta_{v}\right)$ es la transmisión de la atmósfera sobre el camino Sol-superficie, $T\left(\theta_{v}\right)$ es la transmisión de la atmósfera sobre el camino superficie-sensor, $\rho_{\text {Sup }}\left(\lambda, \theta_{S}, \theta_{v}, \phi\right)$ es la reflectancia de la superficie, y $S(\lambda)$ es el cociente de retrodispersión atmosférica que toma en cuenta la reflexión múltiple entre la superficie y la atmósfera. Por otro lado, $T\left(\theta_{S}\right)$ y $T\left(\theta_{v}\right)$ son definidos por las ecuaciones,

$$
\begin{aligned}
& T\left(\theta_{S}\right)=\exp \left[-\frac{1}{\mu_{S}}\left(\tau_{R}+\tau_{a}\right)\right] \\
& T\left(\theta_{v}\right)=\exp \left[-\frac{1}{\mu_{v}}\left(\tau_{R}+\tau_{a}\right)\right] .
\end{aligned}
$$

SARA cuenta con la reflectancia de los aerosoles $\rho_{\text {Aer }}\left(\lambda, \theta_{S}, \theta_{v}, \phi\right)$, que son calculados al sustraer la reflectancia del camino de Rayleigh $\rho_{\text {Ray }}\left(\lambda, \theta_{S}, \theta_{v}, \phi\right)$, y la función de la superficie $\rho_{\mathrm{TOA}}\left(\lambda, \theta_{S}, \theta_{v}, \phi\right)$,

$$
\begin{gathered}
\rho_{\text {Aer }}\left(\lambda, \theta_{S}, \theta_{v}, \phi\right)=\rho_{\text {TOA }}\left(\lambda, \theta_{S}, \theta_{v}, \phi\right)- \\
\rho_{\text {Ray }}\left(\lambda, \theta_{S}, \theta_{v}, \phi\right)-\frac{T\left(\theta_{S}\right) T\left(\theta_{v}\right) \rho_{\text {sup }}\left(\theta_{S}, \theta_{v}, \phi\right)}{1-\rho_{\text {sup }} S(\lambda)}
\end{gathered}
$$

La corrección de los datos de satélite para la dispersión de Rayleigh [20] depende de la determinación de la función fase de Rayleigh 21,22] y el espesor óptico de Rayleigh $\tau_{R}$ [22],

$$
\begin{aligned}
\tau_{R}=\frac{P_{Z}}{P_{0}}\left(8.64 \times 10^{-3}+\right. \\
\left.6.5 \times 10^{-6} z\right) \lambda^{-\left(3.196+0.074+\frac{0.05}{\lambda}\right)}
\end{aligned}
$$

donde $P_{z}$ es la presión ambiente con respecto a la elevación medida en mbar; $P_{0}=1013.25$ mbar, presión al nivel del mar; $z$ la altura del suelo arriba del nivel del mar en $\mathrm{km}$ y $\lambda$ la longitud de onda en $\mu \mathrm{m}$. La reflectancia de la superficie para SARA es obtenida del producto de reflectancia de la superficie diaria MODIS level-2G (MOD09GA) en $500 \mathrm{~m}$ de resolución espacial [23]. Este producto emplea la corrección atmosférica, es decir, la conversión de TOA en la reflectancia de la superficie, para estimar la reflectancia de la superficie MODIS en las bandas del 1 al 7 en el rango de $0.470 \mu \mathrm{m}$ a $2.13 \mu \mathrm{m}$. Nuestro algoritmo SARA usa la longitud de onda del verde, $0.55 \mu \mathrm{m}$, banda 4, de MOD09GA directamente. El método Eddington puede ser usado como una buena aproximación de la razón de retrodispersion atmosférica $S(\lambda)$ [24] para la corrección de la función superficie,

$$
S(\lambda)=\left(0.92 \tau_{R}+(1-g) \tau_{a}\right) \exp \left[-\left(\tau_{R}+\tau_{a}\right)\right] .
$$

La reflectancia de los aerosoles $\rho_{\text {Aer }}\left(\lambda, \theta_{S}, \theta_{v}, \phi\right)$ fue recuperada para la longitud de onda de 0.55 $\mu \mathrm{m}$ por la corrección de la dispersión de Rayleigh $\rho_{\text {Ray }}\left(\lambda, \theta_{S}, \theta_{v}, \phi\right)$ y la función superficie. En la aproximación de dispersión simple, la reflectancia de los aerosoles medida por satélite es proporcional al espesor óptico de los aerosoles $\tau_{a}$, el albedo de dispersión simple $\omega_{0}$ y la función fase de la dispersión de aerosoles $P_{a}[12,13,15,26$,

$$
\rho_{\text {Aer }}\left(\lambda, \theta_{S}, \theta_{v}, \phi\right)=\frac{\omega_{0} \tau_{a, \lambda} P_{a}\left(\theta_{S}, \theta_{v}, \phi\right)}{4 \mu_{S} \mu_{v}} .
$$

Por lo tanto, el AOT $\tau_{a}$ puede ser recuperado al despejarla ecuación (8) como,

$$
\tau_{a, \lambda}=\frac{4 \mu_{S} \mu_{v}}{\omega_{0} P_{a}\left(\theta_{S}, \theta_{v}, \phi\right)} \rho_{\text {Aer }}\left(\lambda, \theta_{S}, \theta_{v}, \phi\right) .
$$

La función fase de dispersión de aerosoles $P_{a}\left(\theta_{S}, \theta_{v}, \phi\right)$ representa la distribución angular de la luz dispersada por las partículas y puede ser determinado usando el método Henyey-Greenstein de un solo término [27],

$$
P_{a}\left(\theta_{S}, \theta_{v}, \phi\right)=\frac{1-g^{2}}{\left[1+g^{2}-2 g \cos (\pi-\theta)\right]^{3 / 2}}
$$

donde $\theta$ es el ángulo de fase de la dispersión [16]. El parámetro asimétrico $g$ indica la dominancia relativa de la dispersión hacia adelante/atrás (forwardscattering/back-scattering) y esto permanece constante para muchos de los modelos de aerosoles [24]. Sustituyendo la ecuación (30) en la ecuación (34) el resultado produce la ecuación (36) donde las tres incógnitas 
$\tau_{a}, \omega_{0}$ y $g$ son mostrados explícitamente. Los valores de $\omega_{0}$ y $g$ obtenidos para el día en el cual la profundidad óptica de los aerosoles, Aerosol Optical Depth-Simplified Aerosol Retrieval Algorithm, AOD-SARA, está siendo recuperado es determinado de un encuentro entre el AOD-SARA como una función de $\omega_{0}$ y $g$, y el AOD-
AERONET dentro de \pm 30 min del tiempo del sobrepaso local del MODIS. Esto es conseguido empíricamente usando el método de iteración de punto fijo, del inglés fixed point iteration, FPI [25] y al variar los valores de $\omega_{0}$ y $g$ hasta que la coincidencia sea obtenida.

$$
\begin{aligned}
\tau_{a, \lambda}=\frac{4 \mu_{S} \mu_{v}}{P_{a}\left(\theta_{S}, \theta_{v}, \phi\right)}\left[\rho_{\mathrm{TOA}}\left(\lambda, \theta_{S}, \theta_{v}, \phi\right)-\rho_{\text {Ray }}\left(\lambda, \theta_{S}, \theta_{v}, \phi\right)\right. & \\
& \left.-\frac{\exp \left[-\left(\tau_{R}+\tau_{a, \lambda}\right) / \mu_{S}\right] \exp \left[-\left(\tau_{R}+\tau_{a, \lambda}\right) / \mu_{v}\right] \rho_{\mathrm{S}}\left(\lambda, \theta_{S}, \theta_{v}, \phi\right)}{1-\rho_{\mathrm{S}}\left(\lambda, \theta_{S}, \theta_{v}, \phi\right)\left(0.92 \tau_{R}+(1-g) \tau_{a, \lambda}\right) \exp \left[-\left(\tau_{R}+\tau_{a, \lambda}\right) / \mu_{\#}\right]}\right]
\end{aligned}
$$

Finalmente, el AOD-SARA ha sido comparado con el AOT-C005 para evaluar la calidad de la recuperación de SARA sobre las nueve posiciones de Hong Kong dentro de los diferentes tipos de cubierta del suelo. Los indicadores estadísticos fueron usados para evaluar las comparaciones del AOT recuperado por el satélite con los heliofotómetros basados en el suelo, es decir, el coeficiente de correlacion $R$, el error de raíz cuadrática media (root mean square error, RMSE), el error absoluto medio (mean absolute error, MAE), el error esperado (expected error, EE) de AOD y la fracción de error esperado (FOE).

\section{Resultados y discusión}

\section{Variabilidad espacial del espesor óptico del aerosol de $550 \mathrm{~nm}$}

La Figura 3 muestra las imágenes de la AOD en la longitud de onda de $550 \mathrm{~nm}$, procedente del sensor MODIS a bordo del satélite TERRA. Estas imágenes tienen una resolución espacial de $1^{\circ} \times 1^{\circ}$ que equivale a $110 \mathrm{~km} \times 110 \mathrm{~km}$ sobre una gran parte de América del Sur, en las latitudes de $4^{\circ} \mathrm{N}$ a $24^{\circ} \mathrm{S}$ y en las longitudes de $83^{\circ}$ a $33^{\circ} \mathrm{W}$, tomadas para el mes de setiembre en un periodo de catorce años, que abarca desde el año 2000 hasta el 2013. En la cuenca amazónica se observa una gran concentración de aerosoles que está en el rango de 0.82-1.0. Existe un crecimiento en la concentración de aerosoles en los años 2004, 2007 y 2010, sobre Brasil, Bolivia hasta Argentina. Uno de los causantes de este aumento en la concentración de los aerosoles en ambos satélites, TERRA y AQUA, se debe al incremento de los focos de incendios forestales en la Amazonía brasileña y boliviana.

Estos incendios forestales son tanto naturales como ocasionados por el hombre. Se provoca incendios con el objetivo de limpiar los terrenos de los bosques y pastizales para ser usados en la ganadería y en la agricultura. Sin embargo, la quema excesiva de vegetación generan muchas partículas contaminantes en la región tropical. El incendio de la vegetación emiten muchos gases como el dióxido de carbono $\mathrm{CO}_{2}$, el monóxido de carbono $\mathrm{CO}$, el metano $\mathrm{CH}_{4}$, óxido nitroso $\mathrm{N}_{2} \mathrm{O}$, los óxidos de nitrógeno $\mathrm{NO}_{x}$ y los aerosoles, y otros hidrocarburos, que tienen un papel importante en la calidad del aire en las ciudades, en la composición química de la atmósfera y el clima.

También los incendios generan la emisión de grandes cantidades de partículas de aerosol con un diámetro de 6 a $10 \mu$ m que interactúan en la formación de la lluvia, este es un tema de gran interés actual debido a la gran incertidumbre acerca de su papel en el sistema de la Tierra, lo que indica que incluso podría tener el efecto contrario al efecto invernadero al reflejar la luz del sol o promover la formación de una mayor cobertura de nubes.

Este proceso de los incendios forestales en Brasil es el origen de los contaminantes en toda la cuenca del Amazonas en el Perú, los contaminantes y aerosoles en el territorio peruano son transportados por los vientos alisios con dirección oeste [6].

\section{Variabilidad temporal del espesor óptico del aerosol de $550 \mathrm{~nm}$}

La Figura 4 muestra los datos mensuales de los AOTs obtenidos por los sensores MODIS abordo de los satélites TERRA (línea negra) y AQUA (línea rojas), sobre el territorio que abarca las latitutes $4^{\circ} \mathrm{N}-24^{\circ} \mathrm{S}$ y las longitudes $83^{\circ} \mathrm{W}-33^{\circ} \mathrm{W}$, observándose una oscilación que varía entre 0.02 y 0.50 , con sus valores máximos entre los meses de agosto o setiembre, durante el período de catorce años que abarca desde el año 2000 hasta el 2013. 


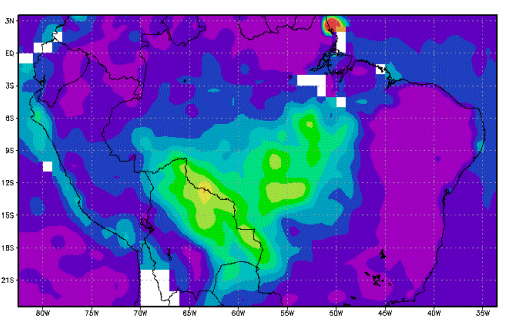

2000

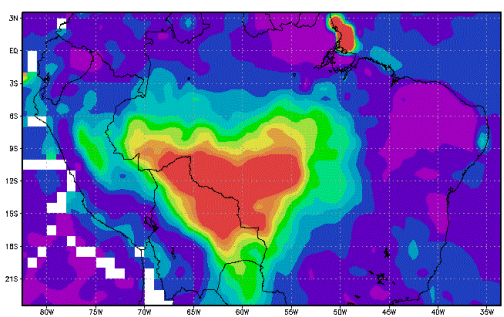

2003

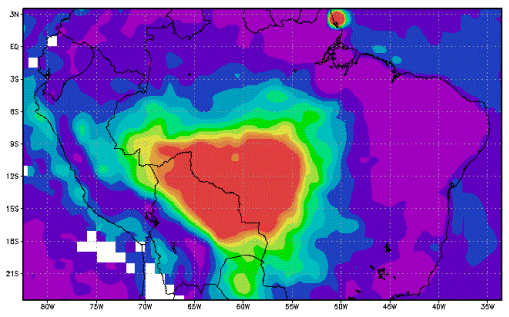

2006

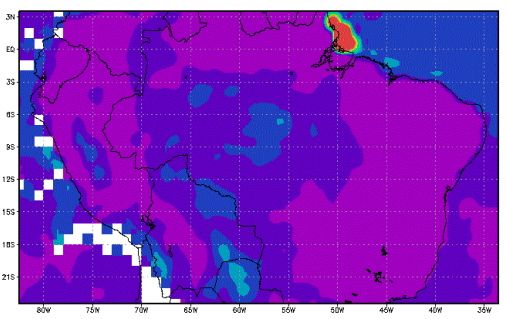

2009

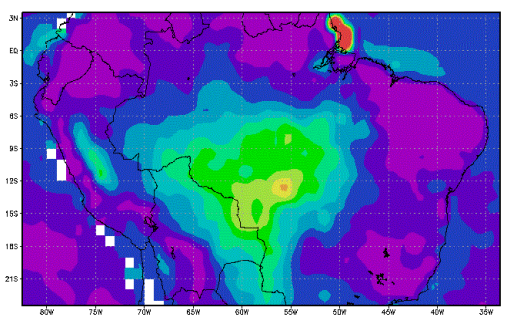

2012

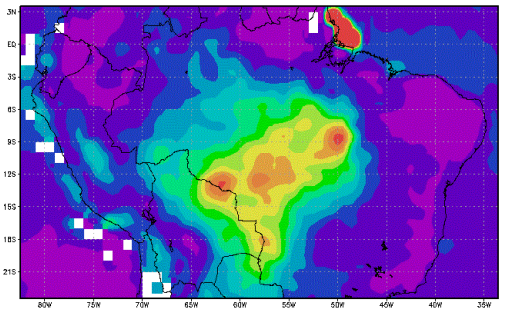

2001

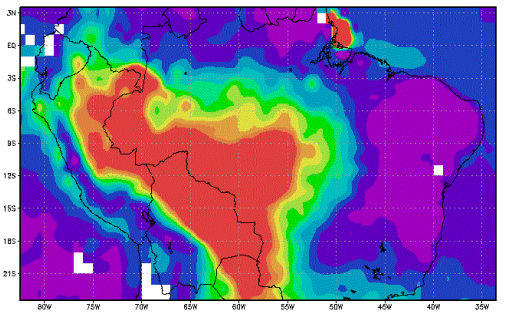

2004

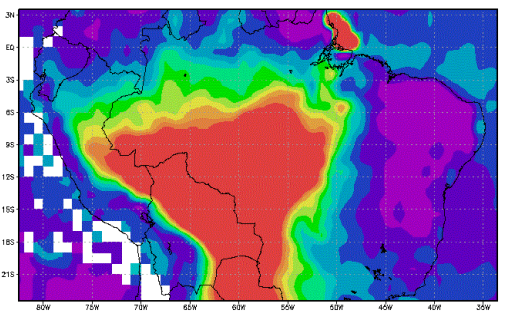

2007

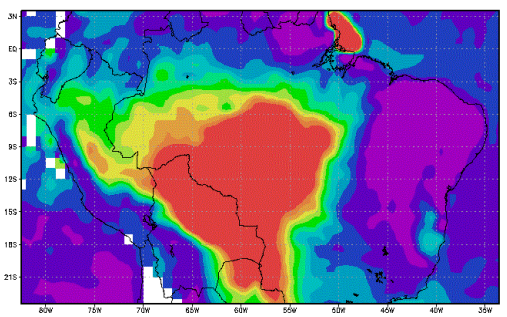

2010

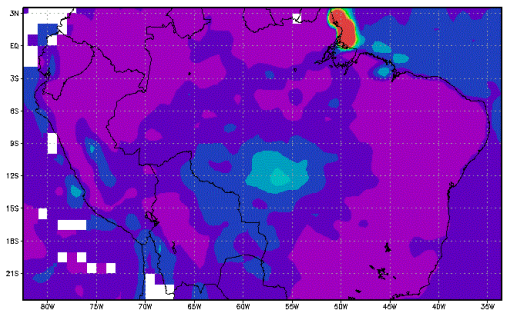

2013

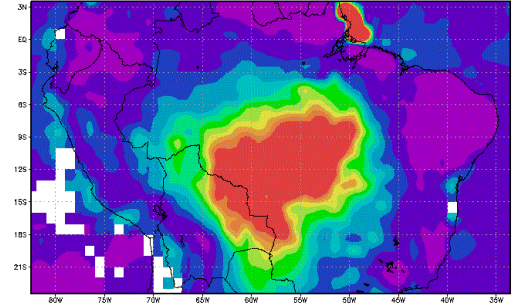

2002

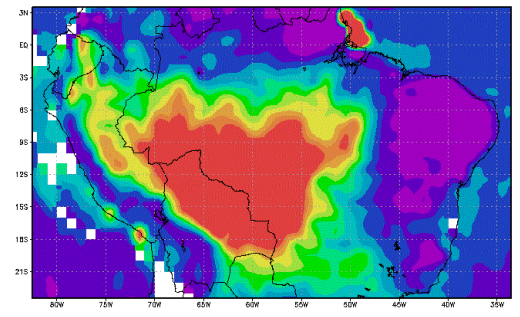

2005

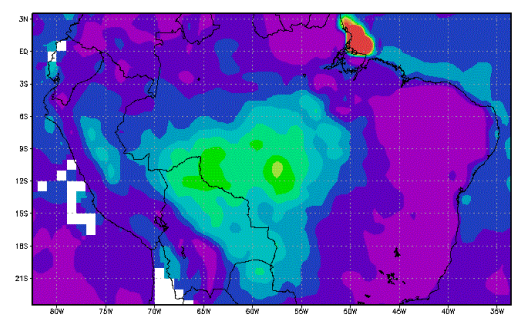

2008

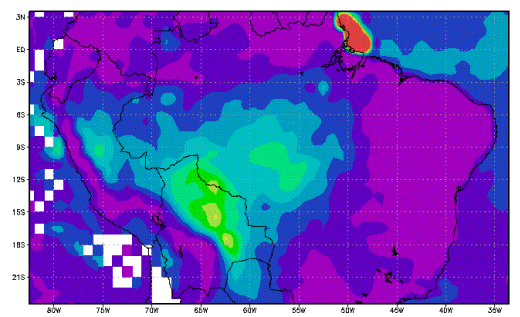

2011

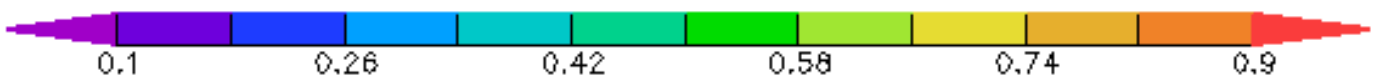

Figura 3: Promedio mensual de la profundidad óptica de los aerosoles en la longitud de onda de $550 \mathrm{~nm}$, procedentes del sensor MODIS a bordo del satélite TERRA, con una resolución espacial de $1^{\circ} \times 1^{\circ}$, de la región comprendida por las latitudes $4^{\circ} \mathrm{N}-24^{\circ} \mathrm{S}$ y las longitudes $83^{\circ} \mathrm{W}-33^{\circ} \mathrm{W}$ para el mes de septiembre desde el año 2000 hasta el 2013. 
Los valores máximos observados en la oscilación durante agosto o setiembre coincide con el inicio de los incendios forestales generados por los agricultores en la región amazónica, que es muy común durante la temporada seca que abarca de agosto a noviembre de cada año. La Amazonía es una zona de quema de vegetación abundante, como parte del cambio de uso del suelo para la explotación agrícola. La diferencia entre los datos del satélite TERRA con el AQUA se basa en la diferencia de tiempo en que pasan estos satélites sobre un mismo lugar.

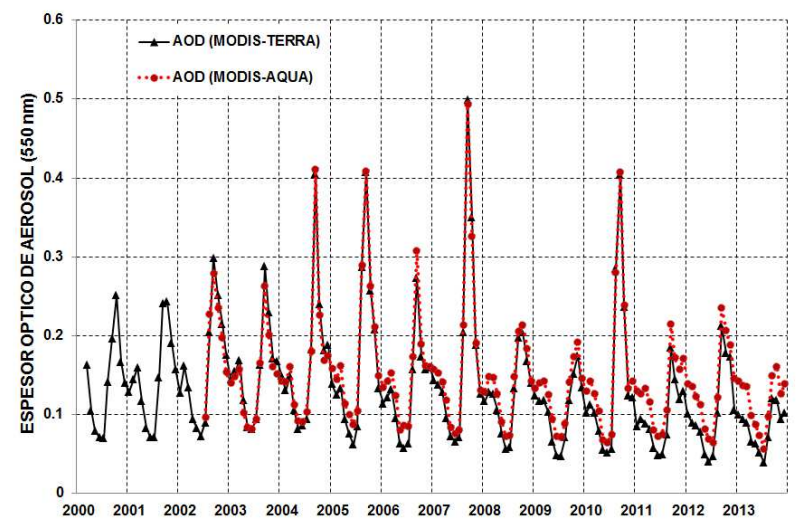

Figura 4: Datos mensuales de la profundidad óptica del aerosol en la longitud de onda de $550 \mathrm{~nm}$, procedente de los MODIS a bordo de los satélites TERRA y AQUA, con una resolución espacial de $1^{\circ} \times 1^{\circ}$, de la región comprendida entre la latitud de $4^{\circ} \mathrm{N}-24^{\circ} \mathrm{S}$ y de longitud $83^{\circ} \mathrm{W}-33^{\circ} \mathrm{W}$ para un periodo de catorce años desde al año 2000 hasta el 2013.

La red AERONET posee seis estaciones en América del Sur cuyos datos son objeto de nuestro interés para nuestro análisis sobre la región del Perú, cuatro ubicadas en Brasil, uno en Bolivia y uno en Chile. Estas estaciones AERONET son (1) la estación de Río Branco que abarca la región entre las latitudes $9^{\circ} \mathrm{S}-10^{\circ} \mathrm{S}$ y las longitudes $68^{\circ} \mathrm{W}-67^{\circ} \mathrm{W}$; (2) la estación de Alta Floresta que abarca la región comprendida entre las latitudes $9^{\circ} \mathrm{S}-10^{\circ} \mathrm{S}$ y las longitudes $57^{\circ} \mathrm{W}-56^{\circ} \mathrm{W}$; (3) la estación de Campo Grande que abarca la región comprendida entre las latitudes $20^{\circ} \mathrm{S}-21^{\circ} \mathrm{S}$ y las longitudes $55^{\circ} \mathrm{W}-54^{\circ} \mathrm{W}$; (4) la estación Ji Paraná SE que abarca la región con latitudes $10^{\circ} \mathrm{S}-11^{\circ} \mathrm{S}$ y las longitudes $62^{\circ} \mathrm{W}-61^{\circ} \mathrm{W}$, todas estas están ubicadas en Brasil; (5) la estación de La Paz, en Bolivia, que comprende la región entre las latitudes $15^{\circ} \mathrm{S}-16^{\circ} \mathrm{S}$ y las longitudes $69^{\circ} \mathrm{W}-68^{\circ} \mathrm{W}$; y (6) la estación de Arica en Chile que comprende la región entre las latitudes $18^{\circ} \mathrm{S}-19^{\circ} \mathrm{S}$ y las longitudes $71^{\circ} \mathrm{W}-70^{\circ} \mathrm{W}$.
En las Figuras 5 y 6 , se observan los datos promedios mensuales de los AOTs obtenidos mediante los sensores MODIS que se encuentran en los satélites TERRA, lado izquierdo de las Fig. 5 y 6 , y AQUA, lado derecho de las Figs 5 y 6 -trazados con líneas negras-, comparado con los datos obtenidos in situ en las seis estaciones de AERONET que comprende, de arriba hacia abajo, las estaciones de Rio Branco, Alta Foresta y Campo Grande en la Fig.5 y las estaciones Ji Paraná SE, La Paz y Arica en la Fig. 6 , trazados con líneas rojas. El periodo de análisis comprende desde el año 2000 hasta el 2013. Los datos de algunas estaciones de AERONET están ausentes durante algún intervalo de tiempo que puede corresponder a problemas de funcionamiento y mantenimiento o también a un recién inicio de operaciones.

Los valores máximos en las oscilaciones de las seis estaciones se observan en las estaciones de Ji Paraná SE y Alta Foresta durante setiembre del 2008 que coinciden con los datos de los sensores MODIS de los satélites TERRA y AQUA. Se observan que los AOTs oscilan alcanzado un máximo entre agosto y setiembre de cada año, descendiendo hasta el mes de noviembre. Estas concentraciones máximas de los AOTs son causadas por la quema de biomasa que se generan mayormente en Brasil y Bolivia. Estos incendios se producen principalmente durante la temporada seca de agosto a noviembre, que también coincide con la capacidad mínima para eliminar los contaminantes vía las precipitaciones que alcanzan sus valores mínimos en la temporada, por tanto, existe una mayor probabilidad de que los contaminantes se dispersen a mayores distancias [1].

Los datos mensuales de los AOTs del sensor MODIS en AQUA adquiridos en el periodo 2002-2013 mostrados con los datos obtenidos in situ por la estación de AERONET en Rio Branco, Brasil, desde el año 2000 al 2011 muestran una oscilación que varía entre 0.0 a 1.9 y 0.0 a 2.0, respectivamente, observándose en ambos casos picos máximos en la temporada seca de agosto a noviembre de cada año. De la misma forma los datos los AOTs del sensor MODIS en TERRA (2000-2013) y del sensor MODIS en AQUA (2002-2013) mostrados con los datos obtenidos in situ por la estación de AERONET en Alta Floresta, Brasil, en el periodo 2000-2012, muestran una oscilación que varía entre 0.0 a 2.0 y 0.0 a 2.1, respectivamente, teniendo un comportamiento similar con la estación Rio Branco. Este comportamiento se debe también a la contaminación atmosférica causa$\mathrm{da}$, principalmente, por los incendios forestales, observándose picos máximos para los años 2005 y 2007. 

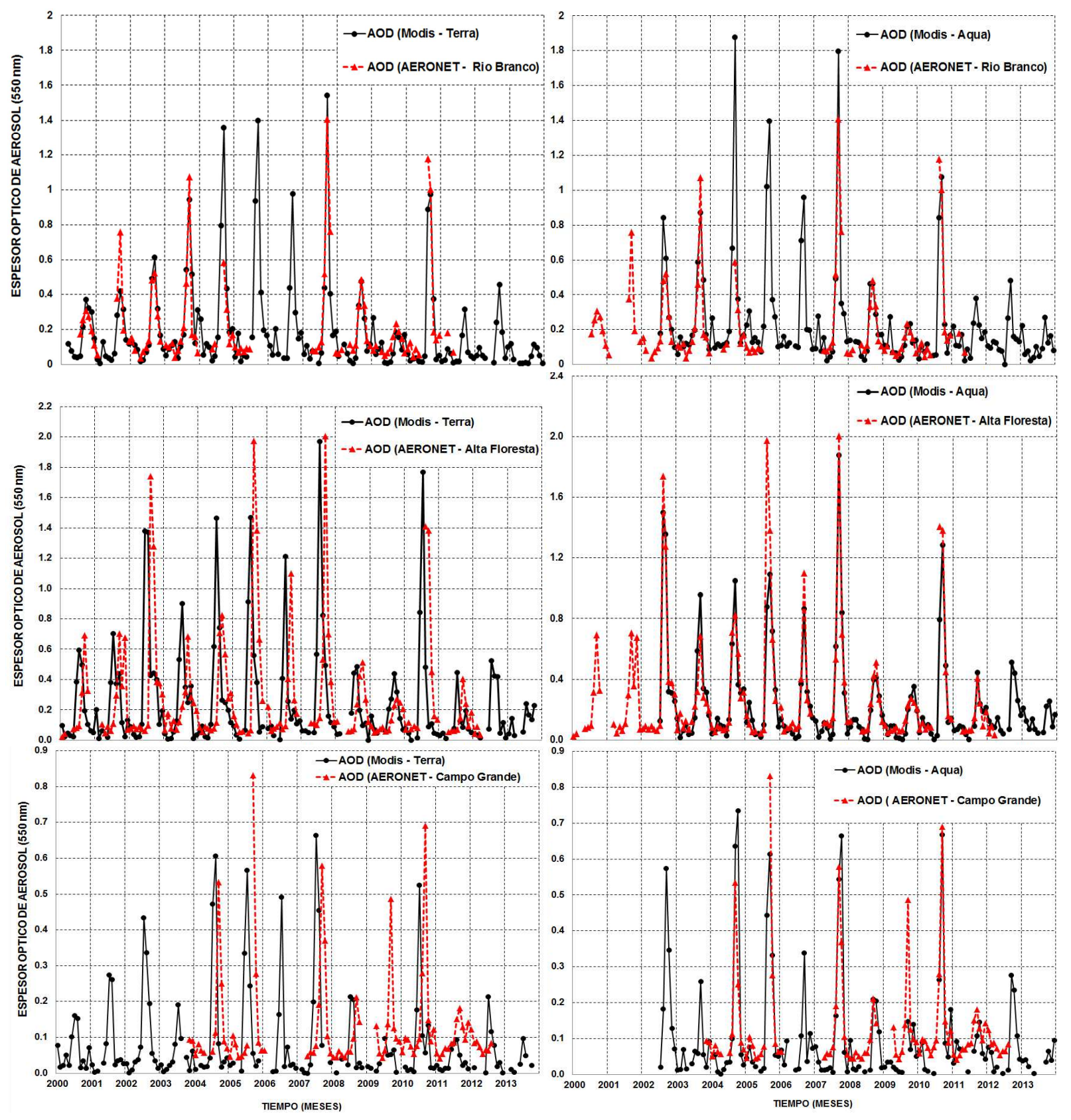

Figura 5: Datos mensuales de la profundidad óptica de los aerosoles en la longitud de onda de $550 \mathrm{~nm}$, procedentes, de arriba hacia abajo, de las estaciones de AERONET en Rio Branco (2000-2011), en Alta Foresta (2000-2012) y en Campo Grande (2003-2011) ubicadas en Brasil comparados con los datos mensuales obtenidos por los sensores MODIS que se encuentran en los satélites TERRA (2000-2013) columna izquierda y AQUA (2002-2013) columna derecha, de las regiones comprendidas por las latitudes $9^{\circ} \mathrm{S}-10^{\circ} \mathrm{S}$ y las longitudes $68^{\circ} \mathrm{W}-67^{\circ} \mathrm{W}$ (Rio Branco), por las latitudes $9^{\circ} \mathrm{S}-10^{\circ} \mathrm{S}$ y las longitudes $57^{\circ} \mathrm{W}-56^{\circ} \mathrm{W}$ (Alta Foresta) y por las latitudes $20^{\circ} \mathrm{S}-21^{\circ} \mathrm{S}$ y las longitudes $55^{\circ} \mathrm{W}-54^{\circ} \mathrm{W}$ (Campo Grande), respectivamente. 

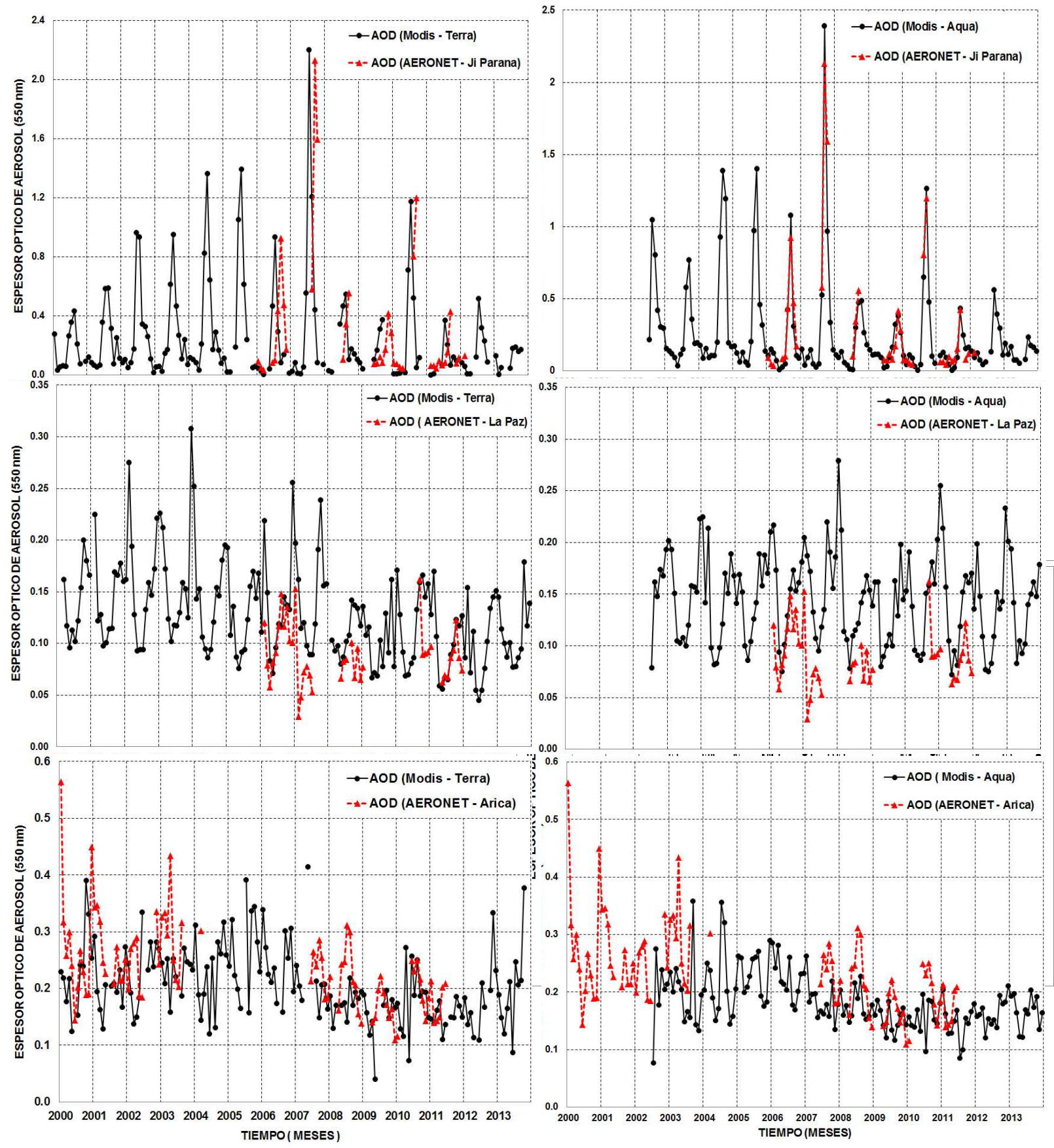

Figura 6: Datos mensuales de la profundidad óptica de los aerosoles en la longitud de onda de $550 \mathrm{~nm}$, procedentes, de arriba hacia abajo, de las estaciones de AERONET en Ji Paraná SE ubicado en Brasil (2006-2010), en La Paz ubicado en Bolivia (2006-2011) y en Arica ubicado en Chile (2000-2013) comparados con los datos mensuales obtenidos por los sensores MODIS ubicados en los satélites TERRA (2000-2013) y AQUA (2002-2013), de las regiones comprendidas por las latitudes $10^{\circ} \mathrm{S}-11^{\circ} \mathrm{S}$ y las longitudes $62^{\circ} \mathrm{W}-61^{\circ} \mathrm{W}$ (Ji Paraná SE), por las latitudes $15^{\circ} \mathrm{S}-16^{\circ} \mathrm{S}$ y las longitudes $69^{\circ} \mathrm{W}-68^{\circ} \mathrm{W}$ (La Paz) y por las latitudes $18^{\circ} \mathrm{S}-19^{\circ} \mathrm{S}$ y las longitudes $71^{\circ} \mathrm{W}-70^{\circ} \mathrm{W}$ (Arica), respectivamente. 
Finalmente, en la Figura 5 (parte inferior), los datos mensuales de los AOTs obtenidos por el sensor MODIS en TERRA (2000-2013)(izquierda) y en sensor MODIS en AQUA (2002-2013)(derecha) mostrados (líneas negras) juntamente con los datos obtenidos in situ por la estación de AERONET en Campo Grande, Brasil, durante el periodo 2003-2011 (líneas rojas), muestran una oscilación que varía entre 0.0 a 0.7 (izquierda); 0.0 a 0.75 (derecha) y 0.0 a 0.85 (in situ), los datos del sensor MODIS con los datos in situ coinciden en su tendencia ascendente máxima en el mes de setiembre, en donde se inicia los incendios forestales en la temporada seca de agosto a noviembre, observando picos máximos para los años 2005, 2007 y 2010.

En la Figura 6, parte superior, se muestran los datos mensuales de los AOTs obtenidos por los sensores MODIS en TERRA (2000-2013) y AQUA (2002-2013) con los datos obtenidos in situ de la estación AERONET de Ji Paraná SE (Brasil) (2006-2010), apreciándose una oscilación que varía entre 0.0 a 2.2 (izquierda); de 0.0 a 2.4 (derecha) y de 0.0 a 2.1 (in situ), con picos máximos en la temporada seca de agosto a noviembre. Para este caso notamos pocos datos obtenidos in situ, pero una alta concentración de aerosoles en el 2007, específicamente en setiembre, en comparación con otros meses y otros años en la Amazonía brasileña.

De la misma forma, en la Figura 6, parte intermedia, se muestran los datos mensuales los AOTs obtenidos por los sensores MODIS en el satélite TERRA (20002013) y en el satélite AQUA (2002-2013) juntamente con los datos obtenidos in situ de la estación de AERONET en La Paz (Bolivia) durante el periodo 2006-2011, mostrando una oscilación que varía entre 0.4 a 0.31 (izquierda); 0.7 a 0.28 (derecha) y 0.3 a 0.16 (in situ). El monitoreo de las áreas quemadas en Bolivia para once años (2000-2010) mostró que se quemaron 22012910 hectáreas en total, de las cuales el 20\% (4 $287512 \mathrm{ha}$ ) corresponden a incendios forestales y la mayor parte de las superficies incendiadas corresponden a pastos y sabanas, superando enormemente las estimaciones realizadas antes de este estudio. En La Paz, la temporada alta de quemas coincide con la época seca que se ve favorecida con los cambios en las condiciones de sequía asociadas al fenómeno de la Oscilación Sur de El Niño (ENSO).

Finalmente, en la Figura 6, parte inferior, se muestran los datos mensuales obtenidos de los AOTs obtenidos por los sensores MODIS en TERRA (2000-2013) y AQUA (2002-2013) juntamente con los datos obtenidos in situ por la estación de AERONET en Arica (Chile) (2000-2011), observándose una oscilación que varía entre 0.0 a 0.4 (izquierda); 0.0 a 0.36 (derecha) y 0.1 a 0.57 (in situ). Se observa que los datos obtenidos in situ tienen una dinámica muy diferente con los datos obtenidos por los sensores MODIS en Terra y en Aqua. Los datos de los AOTs de los MODIS, situada en la misma región que la estación de AERONET en Arica, Chile, muestran valores mínimos en la estación fría de junio a setiembre. En esta época del año se muestra el menor número de observaciones del sensor MODIS por las condiciones nubosas asociadas con las perturbaciones de latitud media que se producen en el invierno austral de junio a agosto. asimismo, Las señales de los sensores MODIS muestra una alta variabilidad en la temporada de verano de noviembre a marzo, que puede explicarse por la contaminación de las señales detectadas por los sensores MODIS [28] con la presencia frecuente de las nubes cirros sobre esta área.

\section{Correlación de los datos de los AOTs pro- cedentes del MODIS y de AERONET}

En las Figuras 7 y 8 , se muestran las correlaciones entre los datos obtenidos de los AOTs mediante los sensores MODIS en TERRA y AQUA con los datos obtenidos in situ en las estaciones de AERONET que se encuentran en Rio Branco, Alta Floresta, Campo Grande y Ji Paraná SE ubicadas en Brasil; en La Paz, Bolivia y en Arica ubicada en Chile. En la parte superior de la Figura 7, corresponde a Rio Branco con una correlación de 0.8800 para TERRA (izquierda) y 0.8365 para AQUA (derecha). En la parte intermedia de la Figura 7, corresponde a Alta Floresta con una correlación de 0.919 para ambos, para TERRA (izquierda) y para AQUA (derecha). La parte inferior de la Figura 7, corresponde a Campo Grande con una correlación de 0.863 para TERRA (izquierda) y de 0.847 para AQUA (derecha).

Para la región de Ji Paraná SE, la parte superior de la Figura 8, muestra la correlación de 0.9833 para TERRA (izquierda) y 0.9585 para AQUA (derecha); mientras que la parte intermedia muestra la correlación de La Paz con TERRA (izquierda) de 0.4367 y con AQUA (derecha) de 0.3734; y finalmente en la parte inferior de la Figura 8, se muestra la correlación de Arica con TERRA (izquierda) de 0.5794 y con AQUA (derecha) de 0.626 . 

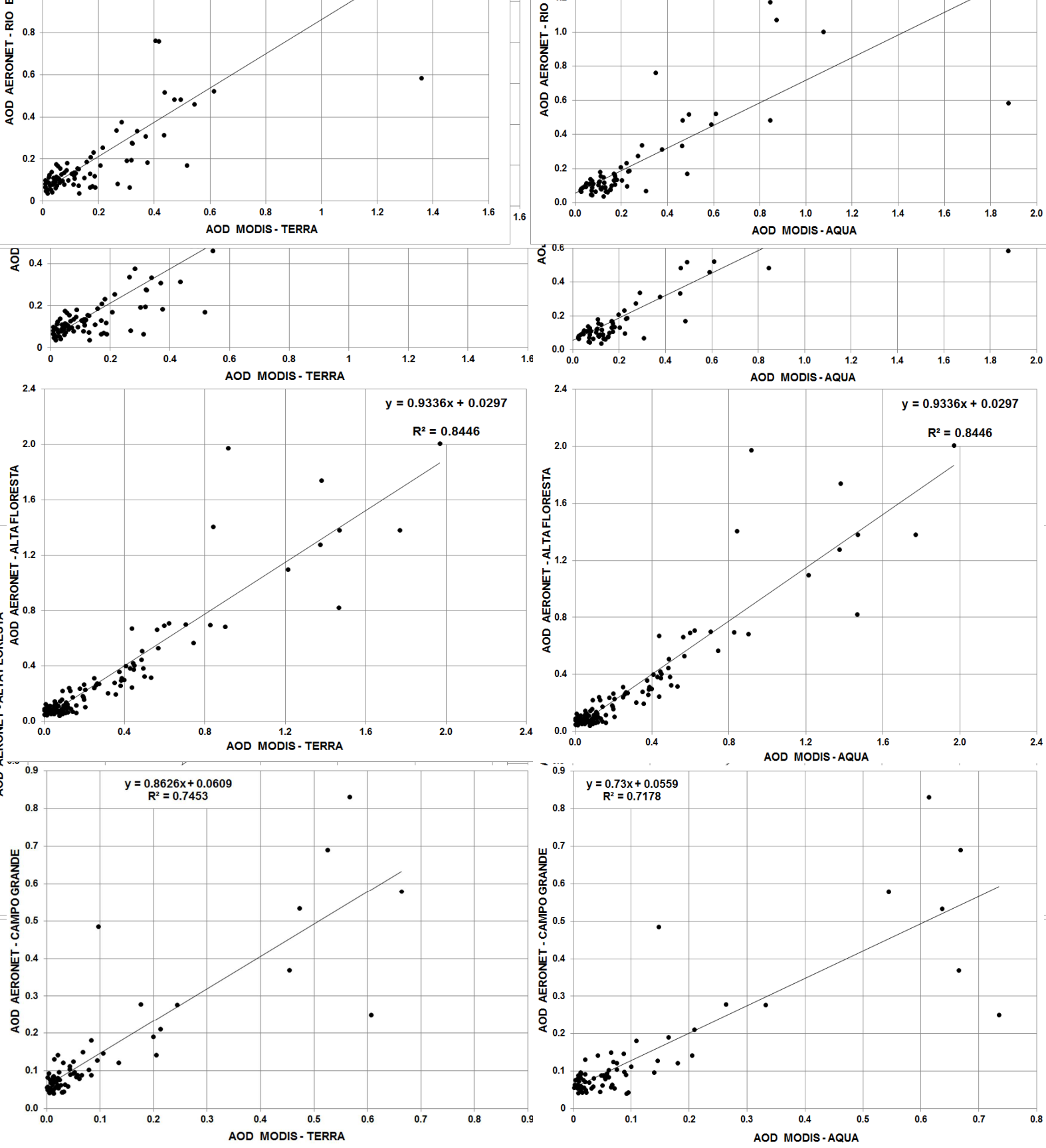

Figura 7: Dispersión de los datos mensuales de las AOD en la longitud de onda de $550 \mathrm{~nm}$, obtenidos en las estaciones de AERONET de Rio Branco (2000-2011) en la figura superior, de Alta Floresta (2000-2012) en la figura intermedia y Campo Grande (2003-2011) en la figura inferior, y dispersión de los datos mensuales detectados por el sensor MODIS en los satélites TERRA (2000-2013) columna izquierda y AQUA (2003-2013) columna derecha en las regiones comprendidas por las latitudes $9^{\circ} \mathrm{S}-10^{\circ} \mathrm{S}$ y las longitudes $68^{\circ} \mathrm{W}-67^{\circ} \mathrm{W}$ (Rio Branco), las latitudes $9^{\circ} \mathrm{S}-10^{\circ} \mathrm{S}$ y las longitudes $57^{\circ} \mathrm{W}-56^{\circ} \mathrm{W}$ (Alta Foresta) y las latitudes $20^{\circ} \mathrm{S}-21^{\circ} \mathrm{S}$ y las longitudes $55^{\circ} \mathrm{W}-54^{\circ} \mathrm{W}$ (Campo Grande), respectivamente. 

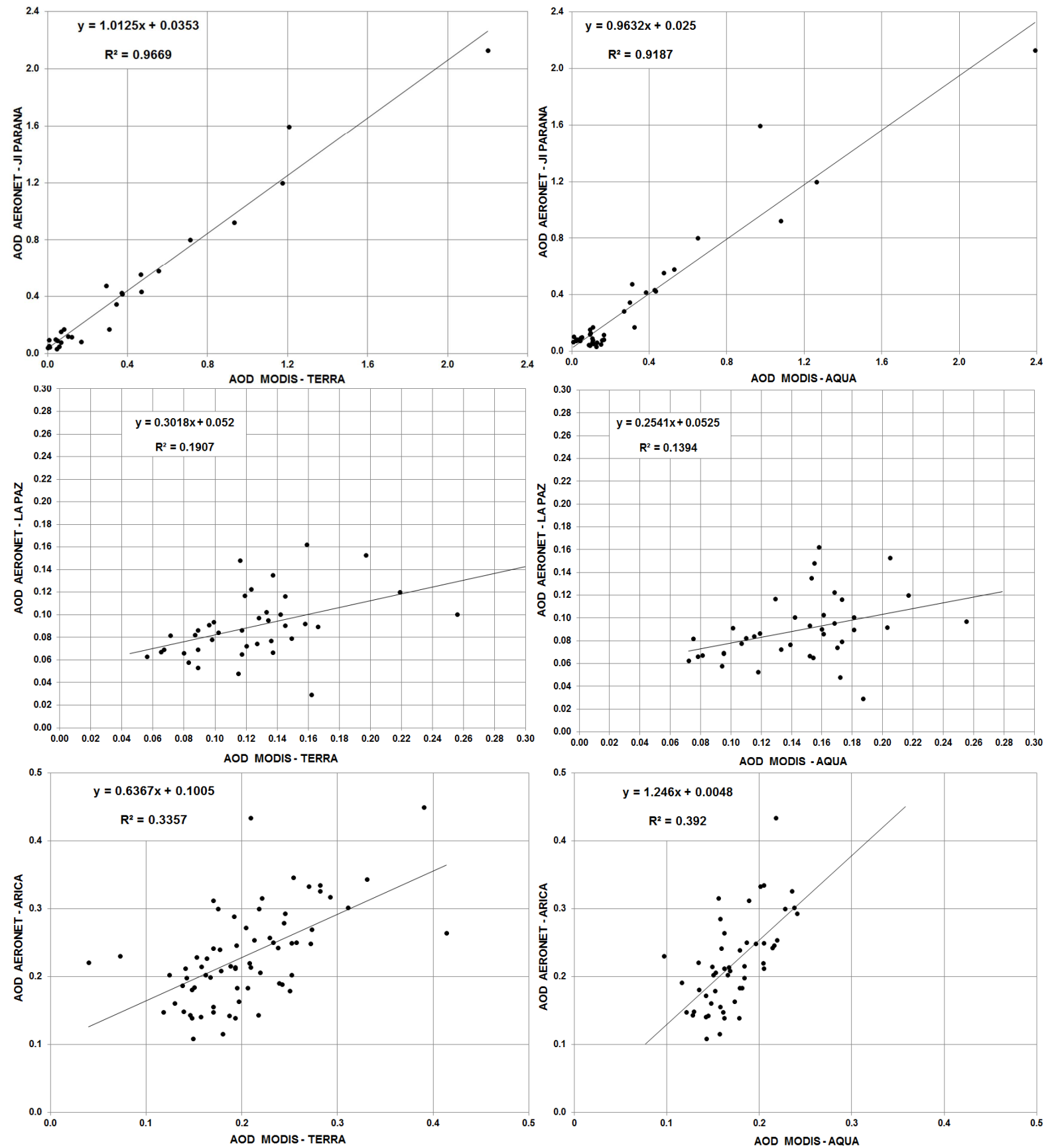

Figura 8: Dispersión de los datos mensuales de las AOD en la longitud de onda de $550 \mathrm{~nm}$, obtenidos en las estaciones de AERONET de Ji Paraná Sé en Brasil (2006-2010) en la figura superior, de La Paz en Bolivia (2006-2011) en la figura intermedia y de Arica en Chile (2003-2011) en la figura inferior, y la dispersión de los datos mensuales detectados por el sensor MODIS en los satélites TERRA (2000-2013) columna izquierda y AQUA (2003-2013) columna derecha en las regiones comprendidas por las latitudes $10^{\circ} \mathrm{S}-11^{\circ} \mathrm{S}$ y las longitudes $63^{\circ} \mathrm{W}-62^{\circ} \mathrm{W}$ (Ji Paraná Sé), las latitudes $16^{\circ} \mathrm{S}-17^{\circ} \mathrm{S}$ y las longitudes $69^{\circ} \mathrm{W}-68^{\circ} \mathrm{W}$ ( La Paz) y las latitudes $18^{\circ} \mathrm{S}-19^{\circ} \mathrm{S}$ y las longitudes $71^{\circ} \mathrm{W}-70^{\circ} \mathrm{W}$ (Arica), respectivamente. 
Las correlaciones entre los datos de las AOTs obtenidos en las estaciones de AERONET ubicadas en Brasil y los datos obtenidos por los sensores MODIS en los satélites TERRA y AQUA son bastante altas si los comparamos con las correlaciones entre los datos de las AOTs obtenidos en las estaciones de AERONET de La Paz en Bolivia y de Arica en Chile y los datos de los MODIS en TERRA y AQUA. Básicamente porque los incendios forestales producidos en la amazonía brasileña es la principal causa en la producción de aerosoles en la atmósfera que en la temporada seca se propaga hacia el oeste mediante los vientos alisios. En La Paz, Bolivia, existen pocos casos de incendios forestales, esto sugiere que existen otras fuentes que originan diferentes tipos de aerosoles que resulta en la extrema variación entre los espesores ópticos de los aerosoles detectados por los MODIS y los detectados por la estación de AERONET en La Paz. Y considerando el resultado de Arica sugiere también la existencia de diferentes tipos de aerosoles puesto que la estación de AERONET en Arica se encuentra muy cerca de los Andes.

\section{Conclusiones}

Las imágenes de las AOTs procedentes de los sensores MODIS (Terra y Aqua) presentan un aumento de la concentración de los espesores ópticos de los aerosoles en la cuenca amazónica. Este aumento se debe a que en Brasil se detectan cientos de focos de incendios forestales en la temporada seca (que abarca de agosto a noviembre, alcanzando un máximo en el mes de setiembre. El incendio forestal controlado es una forma barata de obtener nuevas tierras agrícolas, de eliminar residuos agrícolas y generar un cambio de vegetación. Los datos de las AOTs en las series de tiempo procedentes del sensor MODIS en Terra muestran una dinámica muy similar a los datos de los AOTs procedente del sensor MODIS en Aqua, teniendo picos máximos cada año entre los meses de agosto a noviembre correspondiendo a la temporada más seca de la región. Estos picos máximos corresponden también a la la máxima concentración de aerosoles sobre la cuenca amazónica.
Esta máxima concentración de aerosoles coincide con el inicio de los incendios provocados por los agricultores en la región amazónica, que es muy común durante la temporada seca, lo que también coincide con la menor capacidad para eliminar los contaminantes a través de las precipitaciones que alcanzan sus valores mínimos, por lo cual existe, también, una mayor probabilidad de que los contaminantes se difundan y alcancen mayores distancias.

Los datos de los AOTs obtenidos por las estaciones de AERONET en Brasil, tienen una dinámica muy similar a los datos procedentes de los sensores MODIS en Terra y en Aqua, mostrando picos máximos en la temporada seca (agosto-noviembre) y picos mínimos en los meses de enero a abril, teniendo resoluciones espaciales diferentes. Esta comparación entre dos diferentes métodos de obtener las AOTs es consecuencia de los incendios forestales que se realizan en Brasil y Bolivia, que es una fuente muy importante para el origen de los aerosoles en la tropósfera de la cuenca Amazónica. Los datos AOT-MODIS, situada en la misma región que la estación AERONET (Arica - Chile), muestran valores mínimos en la estación fría (junio-septiembre).

\section{Agradecimientos}

Agradecemos a los miembros del equipo científico de la página web GIOVANNI, ya que los análisis y visualizaciones en esta presentación fue producido con el sistema de datos en línea GIOVANNI, desarrollado y mantenido por el NASA GES DISC. N. R. B. agradece al Vicerrectorado de Investigación de la Universidad Nacional Mayor de San Marcos por la Beca de Tesis de Pregrado 2013-2014. Agradecemos al Proyecto Cátedra CONCYTEC Teledetección de la Desertificación y Sequía por el apoyo financiero para asistir al 19th International Conference on Nucleation and Atmospheric Aerosols. Un agradecimiento muy especial a los miembros del Laboratorio de Teledetección (LABTEL) de la Universidad Nacional Mayor de San Marcos (UNMSM) por las enseñanzas y discusiones realizadas en la preparación de este trabajo.

\section{Referencias}

[1] A. Díaz; Tesis de Doctorado; Universidad La Laguna; España (2006).
[2] A. Esteve; Tesis Doctoral; Departament de Física de la Terra I Termodinámica; Universitat de Valéncia (2011).

[3] Y. J. Kaufman y L. A. Remer; IEEE Trans. Geosci. 
Remote Sensing 32, 672 (1994).

[4] J. Díaz Gonzales, Tesis doctoral, Universidad de la Laguna, España (1999).

[5] R. C. Levy, Doctor of Philosophy Thesis, Maryland(2007).

[6] L. Suárez, L. Castillo, M. Marín, G. Carillo, L. Rimac, J. Pomalaya, R. Menacho; Instituto de Investigación para el Desarrollo Tecnológico, ININTEC, Huancayo, Perú (2006).

[7] G. B. Rybicki, Y. Lightman; Radiative Processes in Astrophysics, Harvard, Smithsonian Center for Astrophysics, Boston (1985).

[8] http://aeronet.gsfc.nasa.gov

[9] http://disc.sci.gsfc.nasa.gov/giovanni.

[10] N. R. Benavente y J. R. Acuña; $19^{\text {th }}$ International Conference, AIP Conference Proceedings 1527(1), 625 (2013).

[11] J. H. Seinfeld y S.N. Pandis, Atmospheric chemistry and physics: From air Pollution to climate change, Cap. XXVII, pp. 1326, John Wiley \& Sons, New York (1998).

[12] A. A. Kokhanovsky y G. De Leeuw, Satellite Aerosol Remote Sensing Over Land, pp. 25-26, Springer, Chichester, UK (2009).

[13] Y. J. Kaufman, D. Tanré et al.; J. Geophys. Res. 102(D14), 17051 (1997).

[14] Y. J. Kaufman, N. Gobron, B. Pinty, J.L. Widlowski y M.M. Verstraete; Geophys. Res. Lett. 29, 2116 (2002).

[15] M. D. King, Y.J. Kaufman, D. Tanre y T. Nakajima; Bull. Am. Met. Soc. 80, 2229 (1999).
[16] R. C. Levy, L.A. Remer, D. Tanré, S. Mattoo, E. F. Vermote y Y. J. Kaufman; J. Geophys. Res. 112, D13211 (2007).

[17] M. Bilal, J. E. Nichol, M. P. Bleiweiss y D. Dubois; Remote Sensing of Environment 136, 135 (2013).

[18] D. Tanré, Y. J. Kaufman et al., J. Geophys. Res. 102(D14), 16971 (1997).

[19] E. F. Vermote, D. Tanré, J. L. Deuze, M. Herman, y J. J. Morcette; IEEE Transactions on Geoscience and Remote Sensing 35, 675 (1997).

[20] D. Antoine y A. Morel; Applied Optics 37, 2245 (1998).

[21] O. Lado-Bordowsky y I. Naour; Internat. J. Infrared and Millimeter Waves 18, 1689 (1997).

[22] S. Liang, Quantitative remote sensing of land surfaces, John Wiley \& Sons, Inc., New Jersey (2005).

[23] E. F. Vermote y S. Kotchenova, J. Geophys. Res. 113, 1 (2008).

[24] D. Tanré, M. Herman, P. Y. Deschamps y A. D. Leffe; Appl. Optics 18, 3587 (1979).

[25] C. T. Kelley, Iterative methods for linear and nonlinear equations; Society for Industrial and Applied Mathematics, SIAM, New York (1995).

[26] H. R. Gordon y M. Wang, Appl. Optics 33, 443 (1994).

[27] H. Rahman, B. Pinty y M. M. Verstraete, J. Geophys. Res. 98, 20791 (1993).

[28] B. - C. Gao, Y. J. Kaufman, D. Tanré y R. -R. Li, Geophys. Res. Lett. 29, 1890, (2002)

[29] A. Oyanadel, D. Painemal, J-F. Léon, I. Chiapello, L. Gallardo; INPE 8, 149 (2006). 